Pacific Journal of Mathematics

CHARACTERIZATION OF GROUP ALGEBRAS IN TERMS OF 


\section{CHARACTERIZATION OF GROUP ALGEBRAS IN TERMS OF THEIR TRANSLATION OPERATORS}

\section{F. P. Greenleaf}

In this paper we will characterize those Banach algebras $A$ which are isometric and isomorphic to the group algebra of some (possibly nonabelian) compact group. The central idea of this characterization is to study the group $G_{\ell}(A)$ of translation operators which act on $A$; here a translation is any linear isometric map of $A$ onto $A$ such that $T(x y)=(T x) y$ for all $x, y \in A$.

We first give a simple characterization of an intermediate class of Banach algebra which includes all group algebras of compact groups and many other closely related algebras. This is the class of QCG algebras: those $A$ isometric and isomorphic to an algebra of the form $A=\overline{\varphi\left(L^{1}(H)\right)} \subset M(H) / N$, where $H$ is a compact group, $N$ a weak $*$ closed two-sided ideal in $M(H)=$ $C(H)^{*}$, and $\varphi: M(H) \rightarrow M(H) / N$ is the canonical homomorphism $(M(H) / N$ is given the quotient norm). This characterization involves the following axiom on $A$.

Axiom $(C A)$ If $\|a\| \leqq 1$ then $L_{a}\left(L_{a}: x \rightarrow a x\right)$ is a strong operator limit of convex sums of translations.

Any QCG algebra has a great number of finite dimensional two-sided ideals; those QCG algebras $A$ which are group algebras are singled out by studying the representations gotten by letting $G_{\ell}(A)$ act on these ideals. Examples are given of QCG algebras which are not the group algebra of any compact group.

In this discussion we assume a knowledge of topological groups as in Weil [16], Chevalley [6], and Pontryagin [13], and of related Banach algebras as found in Loomis [11] and Rickart [14]. All groups will be either compact or locally compact Hausdorff topological groups. If $H$ is such a group, $C_{0}(H)$ is the sup norm algebra of continuous functions vanishing at infinity, and $M(H)=C_{0}(H)^{*}$ is the convolution algebra of bounded regular Borel measures with

$$
\langle\mu * \lambda, \psi\rangle=\int_{H}\left[\int_{H} \psi(s t) d \mu(s)\right] d \lambda(t)
$$

for $\mu, \lambda \in M(H)$ and $\psi \in C_{0}(H)$. As usual, the group algebra $L^{1}(H)$ is the two-sided ideal in $M(H)$ consisting of all measures which are absolutely continuous with respect to left (or right) Haar measure on $H$.

In principle one would expect a characterization of group algebras to be possible, in view of the following result presented in Wendel [17]. 
THEOREM 1.1.1. Two locally compact groups $G$ and $H$ have isometric and isomorphic group algebras $\Leftrightarrow G$ is topologically isomorphic to $H$.

However, examples of nonisomorphic finite groups with isomorphic group algebras are easy to construct (the algebras cannot be isometrically isomorphic); thus, any characterization of group algebras as Banach algebras must rest, in part, on properties which are preserved by isometric isomorphisms but not necessarily under topological isomorphisms. Notice that the translation operators may differ on Banach algebras which are only topologically isomorphic. It is interesting that Wendel makes no use of the natural involution on a group algebra in [17], which suggests that it should be possible to solve the characterization problem without using the additional symmetry provided by an involution.

In order to analyze the characterization problem it has been necessary to introduce auxiliary conditions, such as axioms on the translations, which, while ultimately determined by the norm properties of the algebra, are not easily derived as consequences of a few relations on the algebra and its norm. This is an unfortunate concession to the difficulty of the characterization problem; however, the more direct program has never been carried to a successful conclusion, except in the case of finite or discrete groups. The solutions in these cases will be reviewed later; unfortunately, they cannot be generalized. Recently Rieffel [15] has succeeded in characterizing the group algebras of locally compact abelian groups using auxiliary concepts quite unrelated to the translations studied here.

1.2. Special notation. If $X, Y$ are Banach spaces we denote the strong operator topology on the bounded linear operators $B(X, Y)$ by $(s o)$, and the weak $*$ topology of $X^{*}$ by $(\sigma)=\sigma\left(X^{*}, X\right)$. If $H$ is a locally compact group we can define strong operator topologies $(s o)_{\ell}$ and $(s o)_{r}$ in $M(H)$ by letting $M(H)$ act on the two-sided ideal $L^{1}(H)$ by left or right convolution. We will always set $\Sigma_{X}=\{x \in X ;\|x\| \leqq 1\}$ and let $\mathscr{E}_{X}$ denote the extreme points in $\Sigma_{X}$. If $X$ is just a vector space and $E$ a subset, its linear span is l.s. $[E]$ and its convex span is $\operatorname{co}[E]$. If $(X, \tau)$ is a topological vector space, write $\operatorname{co}[E ;(\tau)]$ for the $(\tau)$ closed convex span of $E \subset X$. We indicate $(\tau)$ convergence of a net $\left\{x_{j}: j \in J\right\}$ to $x \in X$ by writing $x_{j} \stackrel{\tau}{\longrightarrow} x$ or $x \stackrel{\tau}{\longleftarrow} x_{j}$, interchangeably. If $X, Y$ are isometric, isomorphic normed algebras, we will often write this as $X \sim Y$.

\subsection{Properties of group algebras and their translations.}


Definition 1.3.1. If $A$ is a normed algebra, any bounded linear operator $T$ on $A$ with the property $T(x y)=(T x) y(T(x y)=x(T y))$ for $x, y \in A$ is called a left (right) multiplier of $A$. The algebra of all left multipliers on $A$ is denoted by $A_{\ell}^{m}$ and obviously contains, as a left ideal, the algebra of inner left multipliers: $A_{\iota}=\left\{L_{a}: a \in A\right\}$ which arise in the left regular representation of $A$. Similarly define $A_{r}^{m}$ and the left ideal $A_{r}$ of inner right multipliers on $A$. The left (right) translations of $A$ are the groups of all isometric onto left (right) multipliers of $A$ respectively. We denote these groups by $G_{\ell}$ $\left(G_{r}\right)$ and define $G=G_{\iota} \cap G_{r}$.

Since the commutation relation $T(x y)=(T x) y$ is preserved under uniform convergence in $B(A, A), A_{\ell}^{m}$ is a Banach algebra under the operator norm if $A$ itself is a Banach algebra. Similar comments hold for $A_{r}^{m}$.

Definition 1.3.2. An element $x \in A$ in a normed algebra $A$ is left almost invariant if the orbit of $x$ under the action of $G_{\ell}$ lies in a finite dimensional subspace of $A$. Define right almost invariance of $x \in A$ similarly and denote the subalgebras of left (right) almost invariant elements in $A$ by $\mathscr{A}_{\ell}(A)\left(\mathscr{A}_{r}(A)\right)$. Then write $\mathscr{A}(A)=$ $\mathscr{A}_{\ell}(A) \cap \mathscr{A}_{r}(A)$ for the algebra of two-sided almost invariant elements.

In any normed algebra $A$ a left approximate identity is any net $\left\{e_{j}: j \in J\right\}$ such that $\left\|e_{j} a-a\right\| \rightarrow 0$ for any $a \in A$; right approximate identities are defined similarly. An approximate identity is minimal if $\sup \left\{\left\|e_{j}\right\|: j \in J\right\}<\infty$ and $\left\|e_{j}\right\| \rightarrow 1$; we always have $\liminf \left\{\left\|e_{j}\right\|: j \in J\right\} \geqq$ 1 for an approximate identity. If $A$ has a two-sided minimal approximate identity then $\left\|L_{a}\right\|=\|a\|=\left\|R_{a}\right\|$ for $a \in A$ and the left regular representation is an isometric isomorphism so that $A \sim A_{\ell}$; we will frequently identify $A$ with its inner left multipliers. Notice that $R: A \rightarrow A_{r} \subset A_{r}^{m}$ is an isometric anti-isomorphism in this context. If $A$ has a unit $I$ of norm one, then we actually have $A_{\ell}=A_{\ell}^{m}$ and $A_{r}=A_{r}^{m}$.

If $H$ is a locally compact group the relation between $A=L^{1}(H)$, its algebra of multipliers $A_{\ell}^{m}$, and the associated group $G_{\ell}$ of translations has been described in Wendel [18]. We define the homomorphism $L: M(H) \rightarrow A_{\ell}^{m}$ by taking $L(\mu)=L_{\mu}$ where $L_{\mu}(f)=\mu * f$; similarly define anti-homomorphism $R: M(H) \rightarrow A_{r}^{m}$ by taking $R(\mu)=R_{\mu}$ where $R_{\mu}(f)=f * \mu$. These operators include the usual left and right translation operators $L\left(\alpha \delta_{h}\right)$ and $R\left(\alpha \delta_{h}\right)$, where $|\alpha|=1$ and $\delta_{h}$ is the point mass of norm one at $h \in H$. Since there is a two-sided approximate identity of norm one $\left\{f_{j}: j \in J\right\}$ in $L^{1}(H)$ we conclude that $\left\|L_{\mu}\right\|=$ $\|\mu\|=\left\|R_{\mu}\right\|$ so $L$ and $R$ are isometric; in fact we have $f_{j} \stackrel{(\sigma)}{\longrightarrow} \delta_{e}$ which $\Rightarrow \mu * f_{j} \stackrel{(\sigma)}{\longrightarrow} \mu$ in $M(H)$, hence we have 


$$
\begin{aligned}
\|\mu\| \geqq\left\|L_{\mu}\right\| & \geqq \sup \left\{\left\|\mu * f_{j}\right\|: j \in J\right\} \\
& \geqq \lim \sup \left\{\left\|\mu * f_{j}\right\|: j \in J\right\} \geqq\|\mu\|,
\end{aligned}
$$

with similar arguments for $\left\|R_{\mu}\right\|=\|\mu\|$.

THEOREM 1.3.3. Let $H$ be a locally compact group and $A=L^{1}(H)$. Then $A_{\ell}^{m}=\left\{L_{\mu}: \mu \in M(H)\right\}$ and $\left\|L_{\mu}\right\|=\|\mu\|$ for all $\mu \in M(H)$ so that $A_{\ell}^{m}$ can be realized (isometrically and isomorphically) as $M(H)$. In this realization, $G_{\ell}=\left\{L\left(\alpha \delta_{h}\right):|\alpha|=1, h \in H\right\}$; furthermore, if $G_{\ell}$ is given the (so) topology, $G_{\ell}$ is topologically isomorphic to the direct product $S \times H(S$ is the circle group) under the map $\pi:(\alpha, h) \rightarrow$ $L\left(\alpha \delta_{h}\right)$. In fact we have an internal direct product factorization of $G_{\ell}=S I \times \Gamma$, where $I$ is the identity operator, $S I=\{\alpha I:|\alpha|=1\}$, and $\Gamma=\left\{L\left(\delta_{h}\right): h \in H\right\}$ (both $\Gamma$ and $S I$ are (so) closed normal subgroups). The convex span co $\left[G_{\ell}\right]$ is dense in the unit ball $\Sigma_{A} \frac{m}{\zeta}$ in the (so) topology.

Similar results hold for the right multipliers $A_{r}^{m}$, which are realized as $M(H)$ except for the anti-multiplicativity of $R: \mu \rightarrow R_{\mu}$. These facts were either proved by Wendel or follow easily from his results. Notice that $G_{\ell}$ coincides with the usual group of translation operators. In this example, as in any normed algebra, the existence of a minimal two-sided approximate identity implies that each $F \in A_{l}^{m}$ is a (so) limit of inner left multipliers, since $\left\|F\left(e_{j} a\right)-F(a)\right\|=\left\|L_{F e_{j}}(a)-F(a)\right\| \rightarrow 0$.

We will make use of the following properties of a group algebra $A$ of a compact group. There is a two-sided minimal approximate identity in $A$; in fact, since the group is compact, we can find such approximate identities in the center of $A$ (this is proved in the appendix A.1.3). $A$ is a semi-simple algebra (see Rickart [14], Section 2.3 and A.3); indeed, compactness of the group insures that $A$ is a dual Banach algebra with finite dimensional minimal two-sided ideals. Finally, $A$ has at least one nonzero multiplicative linear functional.

REMARK. Dual algebras are defined as in Kaplansky [10]. Later we will use the fact that in a dual algebra with a central minimal approximate identity all the minimal two-sided ideals are finite dimensional; this is proved in the appendix (A.2.1).

The following relations between the group algebra $A$ of a compact group and its group $G_{\ell}$ of left translations will be used as axioms in our program of characterizing group algebras. Each has a left and right handed version. 
Axiom ( $\left.W_{\ell}\right) \quad A$ closed subspace of $A$ is a left ideal $\Leftrightarrow$ the subspace is $G_{\ell}$ invariant.

This property, first proved by Wiener for the group algebra $L^{1}(-\infty, \infty)$, on a normed algebra insures existence of enough translations to distinguish closed left ideals from other closed subspaces in $A$,

Axiom $\left(A I_{\ell}\right)$ The left almost invariant elements $\mathscr{A}_{\ell}(A)$ are norm dense in $A$.

The two-sided version of this axiom says that $\mathscr{A}(A)$ is norm dense in $A$. In a compact group algebra $A$ the subalgebras of left, right, and two-sided almost invariant elements coincide and are isomorphic to a direct sum of (possibly infinitely many) full finite dimensional matrix algebras over the complex numbers (see Loomis [11], 39D for a detailed study). This axiom limits the size of $G_{\ell}$ (if $G_{\ell}$ is large it is difficult for $a \in A$ to be left almost invariant); for example if $R^{1}$ is the real line and $A=C_{0}\left(R^{1}\right)$, then $G_{\ell}$ is identified with all continuous unimodular functions on $R^{1}$ and $f(x) \equiv 0$ is the only almost invariant element in $A$.

These axioms will eventually be replaced in our discussion by requiring that the algebra $A$ under consideration be a dual Banach algebra with central minimal approximate identity. The following convex approximation property; implicit in Wendel [18], cannot be replaced by such conditions and is really the axiom on which our characterization rests. Clearly it is suggested by the Krein-Milman Theorem; these approximation properties in the $(s o)$ topology are also discussed in Section 1 of Greenleaf [8].

Axiom $\left(C A_{\ell}\right)$ Every inner left multiplier $L_{a}$, for $\|a\| \leqq 1$ in $A$, is the strong operator limit of convex sums of left translations.

Here is another formulation of this axiom.

LeMma 1.3.4. If $A$ is a normed algebra with minimal two-sided approximate identity, then $\left(C A_{\ell}\right)$ holds $\Leftrightarrow$ the convex span $\operatorname{co}\left[G_{\ell}(A)\right]$ is (so) dense in the unit ball $\Sigma_{\Delta}^{m}$.

Proof. Since $\left\|L_{a}\right\|=\|a\|$ for $a \in A$ (use the approximate identity) the implication $(\Leftrightarrow)$ is clear. Conversely if the $(s o)$ closure of $\operatorname{co}\left[G_{\ell}\right]$ includes $\left\{L_{x}: x \leqq 1\right\}$, which is (so) dense in $\Sigma_{A_{l}^{m}}$ due to minimality of our approximate identity, it follows that $\operatorname{co}\left[G_{\ell}:(s o)\right]=\Sigma_{A_{\ell}^{m}}$. 
REMARK. When $H$ is compact we will sacrifice grammar for conciseness and refer to $L^{1}(H)$ as a "compact group algebra".

1.4. QCG algebras and their significance. If $A$ is a Banach algebra, we will show axiom $\left(A I_{\ell}\right)$ insures that $\left(G_{\ell},(s o)\right)$ is a compact topological group; since $\left(A I_{\ell}\right)$ holds in any semi-simple dual Banach algebra with finite dimensional minimal two-sided, this is not an uncommon state of affairs. It is then natural to try to represent $A$ in terms of $L^{1}\left(G_{\ell}\right)$ or $M\left(G_{\ell}\right)$. If $A$ has a minimal two-sided approximate identity, satisfies axiom $\left(A I_{\ell}\right)$, and has the convex approximation property: Axiom $\left(C A_{\ell}\right)$, then we will show that $A_{\ell}^{m} \sim M\left(G_{\ell}\right) / N$, where $N$ is a $(\sigma)$ closed two-sided ideal, and that $A \sim \overline{\varphi\left(L^{1}\left(G_{\ell}\right)\right)} \subset$ $M\left(G_{\ell}\right) / N$ in this representation, where $\varphi: M\left(G_{\ell}\right) \rightarrow M\left(G_{\ell}\right) / N$ is the canonical homomorphism and $M\left(G_{\ell}\right) / N$ is given the quotient norm. This gives half of the characterization of QCG algebras.

DEFINITION 1.4.1. Let $H$ be a compact group, $N \subset M(H)$ any ( $\sigma)$ closed two-sided ideal, and $\varphi: M(H) \rightarrow M(H) / N$ the canonical homomorphism. If $M(H) / N$ is given the quotient norm, then $A=$ $\overline{\varphi\left(L^{1}(H)\right)}$ is a Banach algebra; an abstract Banach algebra is called a $Q C G$ algebra if it is isometric and isomorphic to an algebra of this type for some compact group $H$ and some $(\sigma)$ closed two-sided ideal $N \subset M(H)$.

If $A$ is a QCG algebra, we will see that most properties of compact group algebras also hold for $A$. On the other hand we will exhibit QCG algebras which satisfy all properties set forth in Section 1.3 and still fail to be group algebras. The QCG algebras are plentiful and their many similarities with group algebras may account for some of the difficulty of the group algebra characterization problem.

1.5. The finite characterization problem. The characterization problem for finite groups is solved below. Here $A=L^{1}(H)=M(H)$, there exists a unit of norm one, and there is no difficulty introducing the correct topology on $G_{\ell}(A)$. With a little effort the first characterization can be adapted to identify the group algebra of any discrete group; we leave this to the reader.

THEOREM 1.5.1. A finite dimensional normed algebra $A$ with identity of norm one is a group algebra $\Leftrightarrow$

(1) The extreme points $\mathscr{E}_{A}$ in $\Sigma_{A}$ form a group.

(2) The algebra has a nonzero multiplicative linear functional.

(3) If $\left\{g_{1} \cdots g_{n}\right\}$ are pair wise linearly independent points in $\mathscr{E}_{A}$, then $\left\|\sum_{i=1}^{n} \alpha_{i} g_{i}\right\|=\sum_{i=1}^{n}\left|\alpha_{i}\right|$. 
Proof. It suffices to prove $(\Leftarrow)$. Notice $A_{\ell}^{m} \sim A$ under the left regular representation. For any normed algebra $A, G_{\ell}(A)=\mathscr{E}_{\Lambda_{\ell}^{m}}$ if the extreme points $\mathscr{E}_{\Lambda^{m}}$ form a group (see A.3.1 for verification) so we identify $G_{\ell}=\mathscr{E}_{A}$. If $\Psi$ is the functional in (2) and $\Gamma=$ $\left\{g \in G_{\ell}: \Psi(g)=1\right\}$, then $\Gamma$ is a normal subgroup in $G_{\ell}$, as is $S I=$ $\{\alpha I:|\alpha|=1\}$, and we have an internal direct product factorization $G_{\ell}=S I \times \Gamma$. Give $\Gamma$ the discrete topology and define $T: M(\Gamma) \rightarrow A$ such that $T\left(\sum_{i=1}^{n} \alpha_{i} \delta_{\gamma_{i}}\right)=\sum_{i=1}^{n} \alpha_{i} \gamma_{i}$ (notice that the $\gamma \in \Gamma$ are pairwise linearly independent, and (3) $\Rightarrow$ the elements of $\Gamma$ are linearly independent; hence $\Gamma$ is finite). This is an isometric onto mapping; in fact, $\left\|\sum_{i=1}^{n} \alpha_{i} \delta_{\gamma_{i}}\right\|=$ $\sum_{i=1}^{n}\left|\alpha_{i}\right|=\left\|\sum_{i=1}^{n} \alpha_{i} \gamma_{i}\right\|$ from (3), and clearly

$$
\Sigma_{A}=\operatorname{co}\left[\mathscr{E}_{A}\right]=\operatorname{co}\left[T\left(\mathscr{E}_{M(T)}\right)\right]=T\left(\operatorname{co}\left[\mathscr{E}_{M(\Gamma)}\right]\right)=T\left(\Sigma_{M(\Gamma)}\right)
$$

from finite dimensionality. Direct computation shows that $T(\mu * \lambda)=$ $T(\mu) \cdot T(\lambda)$.

CoRollary 1.5.2. A finite dimensional algebra $A$ can be given a group algebra norm $\Leftrightarrow$ it has at least one linear basis $\mathscr{P}=\left\{x_{1}, \cdots, x_{n}\right\}$ which forms a group under multiplication. If $\mathscr{X}$ is any such basis the (unique) group algebra norm associated with it is such that $S \mathscr{X}=\{\alpha x:|\alpha|=1, x \in \mathscr{X}\}$ coincides with the extreme points $\mathscr{E}_{A}$, and $\Sigma_{A}=\operatorname{co}[S \mathscr{X}]$ in $A$.

Proof. If $x=\sum_{i=1}^{n} \alpha_{i} x_{i}$ in $A$, take $\|x\|=\sum_{i=1}^{n}\left|\alpha_{i}\right|$.

\section{Characterization of QCG algebras.}

2.1. Concrete representation theorems for Banach algebras. First we display various conditions which insure that $\left(G_{\ell},(s o)\right)$ is a compact topological group.

THeOREM 2.1.1. If $A$ is a Banach algebra and the left almost invariant elements are norm dense in $A$ then $\left(G_{\ell}(A),(s o)\right)$ is a compact topological group. A similar result holds for $\left(G_{r}(A),(s o)\right)$ if axiom $\left(A I_{r}\right)$ holds.

Proof. We present the left-handed case. For a Banach algebra $A,\left(G_{\ell}(A),(s o)\right)$ is always a Hausdorff topological group (see verification in appendix (A.3.1)). The rest of the proof is given in two lemmas.

LEMmA 2.1.2. If $A$ is a Banach algebra, let $O_{a}=\left\{g(a): g \in G_{\ell}\right\}$ for $a \in A$, and consider the conditions:

(1) $\left(G_{\ell}(A),(s o)\right)$ is compact 
(2) $O_{a}$ has compact norm closure in $A$ for any $a \in A$.

(3) The right multiplication $R_{a}$ is a compact operator for any $a \in A$.

Then $(2) \Rightarrow(1)$, and if $A$ has a minimal two-sided approximate identity we also have $(3) \Rightarrow(2)$.

Proof. If $\left\{e_{j}: j \in J\right\}$ is the approximate identity, then $F \in A_{\ell}^{m} \Rightarrow$ $\left\|F\left(e_{j}\right) a-F(a)\right\| \rightarrow 0$, so that $O_{a} \subset \Sigma_{A^{m}}(a) \subset \overline{\Sigma_{A} \cdot a}$, which is norm compact; hence $(3) \Rightarrow(2)$. For $(2) \Rightarrow(1)$ we consider the product space $Q=\Pi\left\{\left(O_{a}\right)^{-}: a \in A\right\}$ with the Cartesian product topology (hence $Q$ is compact). Define maps $\tau$ and $\omega$ mapping $G_{/}$into $Q$ by requiring that $\pi_{a}(\tau(g))=g(a)$ and $\pi_{a}(\omega(g))=g^{-1}(a)$ in $O_{a}$ for $g \in G_{\ell}, a \in A\left(\pi_{a}\right.$ is the projection of $Q$ onto $\left.\left(O_{a}\right)^{-}\right)$. If $\left\{g_{j}: j \in J\right\}$ is a net in $G_{\ell}$ there must exist a subnet $\left\{g_{j(k)}: k \in K\right\}$ such that both $\left\{\tau\left(g_{j(k)}\right): k \in K\right\}$ and $\left\{\omega\left(g_{j(k)}\right): k \in K\right\}$ are convergent in $Q$. This happens $\Leftrightarrow$ we have $\left\|g_{j(k)}(a)-\pi_{a}(x)\right\|=\left\|\pi_{a}\left(\tau\left(g_{j(k)}\right)\right)-\tau_{a}(x)\right\| \rightarrow 0$ and $\left\|g_{j(k)}^{-1}(a)-\pi_{a}(y)\right\|=$ $\left\|\pi_{a}\left(\omega\left(g_{j(k)}\right)\right)-\pi_{a}(y)\right\| \rightarrow 0$ for $a \in A$, where $x$ and $y$ in $Q$ are the respective limits of these nets.

Define maps $g_{\infty}(a)=\pi_{a}(x)=\lim \left\{g_{j(k)}(a): k \in K\right\}$ and $h_{\infty}(a)=\pi_{a}(y)=$ $\left.\lim \left\{g_{j(k)}\right)^{-1}(a): k \in K\right\}$ for all $a \in A$. We assert that $g_{\infty}, h_{\infty}$ are isometries in $A_{\ell}^{m}$; it suffices to prove this for $g_{\infty}$. Linearity of $g_{\infty}$ is easy to check; furthermore, $g_{\infty}(a b)=\lim \left\{g_{j(k)}(a b): k \in K\right\}=\lim \left\{g_{j(k)}(a) \cdot b\right\}=$ $\lim \left\{g_{j(k)}(a)\right\} \cdot b=g_{\infty}(a) \cdot b$, and $\left\|g_{\infty}(a)\right\|=\lim \left\{\left\|g_{j(k)}(a)\right\|: k \in K\right\}=\|a\|$. The maps $g_{\infty}, h_{\infty}$ are onto if $g_{\infty} \circ h_{\infty}=h_{\infty} \circ g_{\infty}=I$ (identity map on $A$ ), and this follows since $\left\|g_{\infty}\left(h_{\infty}(a)\right)-a\right\|=\left\|g_{\infty}\left(h_{\infty}(a)\right)-g_{j(k)}\left(h_{\infty}(a)\right)\right\|+$ $\left\|g_{j(k)}\left(h_{\infty}(a)\right)-g_{j(k)}\left(g_{j(k)}{ }^{-1}(a)\right)\right\|=\left\|g_{\infty}\left(h_{\infty}(a)\right)-g_{j(k)}\left(h_{\infty}(a)\right)\right\|+\| h_{\infty}(a)-$ $g_{j(k)}{ }^{-1}(a) \| \rightarrow 0$ for $a \in A$, with a similar argument for $h_{\infty} \circ g_{\infty}=I$. Thus $g_{j(k)} \stackrel{(s o)}{\longrightarrow} g_{\infty}$ in $G_{l}$ and we have demonstrated $(2) \Rightarrow(1)$.

Lemma 2.1.3. If $A$ is a Banach algebra such that $\mathscr{A}_{\ell}(A)$ is norm dense in $A$, then the norm closure $\left(O_{a}\right)^{-}$of $O_{a}=\left\{g(a): g \in G_{\ell}\right\}$ is always norm compact in $A$. A similar right-handed result holds.

Proof. If $a \in \mathscr{A}_{\ell}$, clearly $\left(O_{a}\right)^{-}$is norm compact. If $a \in A$ select $\left\{a_{n}: n=1,2 \cdots\right\} \subset \mathscr{A}_{\ell}$ such that $\left\|a-a_{n}\right\|<1 / 2^{n}$ and give $Q=\Pi_{n=1}^{\infty}\left(O_{a_{n}}\right)^{-}$ the product topology (so $Q$ is compact). Define $\tau: g \rightarrow\left\{g\left(a_{n}\right)\right\} \in Q$; it is easy to see that $O_{a}^{-}$is norm compact $\Leftrightarrow$ corresponding to any net $\left\{g_{j}(a)=z_{j}: j \in J\right\}$ in $O_{a}$, there exists a subnet $\left\{g_{j(k)}(a): k \in K\right\}$ which is norm convergent in $A$ (its limit is in $\left.\left(O_{a}\right)^{-}\right)$. There is a subnet $\left\{g_{j(k)}\right\}$ such that $\tau\left(g_{j(k)}\right) \rightarrow x \in Q$ in the product topology; this means that $g_{j(k)}\left(a_{n}\right) \stackrel{(\text { norm })}{\longrightarrow} x_{n}=\pi_{n}(x)$ for $n=1,2 \cdots . \quad$ Now $\left\|\pi_{n+1}(x)-\pi_{n}(x)\right\| \leftarrow$ $\left\|g_{j(k)}\left(a_{n+1}\right)-g_{j(k)}\left(a_{n}\right)\right\|=\left\|a_{n+1}-a_{n}\right\| \leqq 1 / 2^{n}$, so $\left\{\pi_{n}(x)\right\}$ is Cauchy and converges in norm to some $x^{*} \in A$. This means that 


$$
\begin{aligned}
& \left\|g_{j(k)}(a)-x^{*}\right\| \leqq\left\|g_{j(k)}(a)-g_{j(k)}\left(a_{n}\right)\right\|+\left\|g_{j(k)}\left(a_{n}\right)-x_{n}\right\|+\left\|x_{n}-x^{*}\right\| \\
& \leqq 1 / 2^{n-1}+\left\|g_{j(k)}\left(a_{n}\right)-x_{n}\right\|+\left\|x_{n}-x^{*}\right\| \quad \text { for } n=1,2 \cdots ;
\end{aligned}
$$

clearly then $\left\|g_{j(k)}(a)-x^{*}\right\| \rightarrow 0$ as required.

THEOREM 2.1.4. Let $A$ be a Banach algebra with minimal twosided approximate identity, and assume that $A$ satisfies axioms $\left(A I_{\ell}\right)$ and $\left(C A_{\ell}\right)$. Then there exists a compact topological group $H$ and ( $\sigma)$ closed two-sided ideal $N \subset M(H)$ such that $A_{l}^{m}$ is isometric and isomorphic to $M(H) / N$ and $A$ is isometric and isomorphic to the algebra $\overline{\varphi\left(L^{1}(H)\right)}$ in $M(H) / N$, where $\varphi: M(H) \rightarrow M(H) / N$ is the canonical homomorphism and $M(H) / N$ is given the quotient norm.

Proof. From 2.1.1, $\left(G_{\ell},(s o)\right)$ is compact. Because the functions $g \rightarrow g(a)$ are (so) continuous on $G_{\ell}$ for $a \in A$, we can define the vector valued integrals $T \mu(a)=\int_{\sigma_{\ell}} g(\alpha) d \mu(g)$ such that

$$
\left\langle T \mu(a), a^{*}\right\rangle=\int_{a_{\ell}}\left\langle g(a), a^{*}\right\rangle d \mu(g)
$$

for $\mu \in M\left(G_{\ell}\right), a \in A, a^{*} \in A^{*}$. As indicated in Bourbaki [5], p. 80-82, each functional on $A^{*}$ defined by this relation corresponds to an element of $A$. Thus we have defined an operator $T \mu: A \rightarrow A$ such that $\|T \mu\| \leqq\|\mu\|$. To see that $T \mu \in A_{\ell}^{m}$, let $R_{b}$ be the right multiplication by $b \in A$; then

$$
\begin{aligned}
\left\langle T \mu(a b), a^{*}\right\rangle & =\int_{a_{\ell}}\left\langle g(a b), a^{*}\right\rangle d \mu(g) \\
& =\int_{a_{\ell}}\left\langle(g(a)) b, a^{*}\right\rangle d \mu(g) \\
& =\int_{a_{\ell}}\left\langle g(a), R_{b}^{*} a^{*}\right\rangle d \mu(g) \\
& =\left\langle T \mu(a), R_{b}^{*} a^{*}\right\rangle=\left\langle R_{b} T \mu(a), a^{*}\right\rangle=\left\langle T \mu(a) \cdot b, a^{*}\right\rangle
\end{aligned}
$$

as required. Furthermore, $T: M\left(G_{\ell}\right) \rightarrow A_{\ell}^{m}$ is a homomorphism since

$$
\begin{aligned}
\left\langle T \mu(T \lambda(a)), a^{*}\right\rangle & =\left\langle T \lambda(a),(T \mu)^{*} a^{*}\right\rangle \\
& =\int_{a_{\ell}}\left\langle g(a),(T \mu)^{*} a^{*}\right\rangle d \lambda(g) \\
& =\int_{a_{\ell}}\left\langle T \mu g(a), a^{*}\right\rangle d \lambda(g) \\
& =\int_{a_{\ell}}\left[\int_{a_{\ell}}\left\langle h g(a), a^{*}\right\rangle d \mu(h)\right] d \lambda(g) \\
& =\int_{a_{\ell}}\left\langle t(a), a^{*}\right\rangle d \mu * \lambda(t)=\left\langle T(\mu * \lambda) a, a^{*}\right\rangle .
\end{aligned}
$$


If $N=\operatorname{Ker} T$, then $N$ is a two-sided ideal in $M\left(G_{\ell}\right)$.

The map $T:\left(M\left(G_{\ell}\right),(\sigma)\right) \rightarrow\left(A_{\ell}^{m},(s o)\right)$ is continuous on norm bounded sets; it suffices to verify this on $\Sigma_{M(G /)}$. Let $\mu_{j} \stackrel{(\sigma)}{\longrightarrow} \mu$ in $\Sigma_{M(\theta /)}$. First notice that $\Omega=\operatorname{co}\left[G_{\ell}:(s o)\right]$ is compact in $\left(A_{\zeta}^{m},(s o)\right)$, since the (so) convex closure of an (so) compact set in $B(A, A)$ is again (so) compact (see A.4.2 in appendix); then $T \mu \in \Omega$ whenever $\mu \in \Sigma_{M(G \ell)}$. Indeed, if $\lambda_{j} \in \operatorname{co}\left[\mathscr{E}_{M\left(G_{\ell}\right)}\right]$ is a net such that $\lambda_{j} \stackrel{(\sigma)}{\longrightarrow} \mu$, then $T \lambda_{j} \in \Omega$ and there is an $(s o)$ convergent subnet $T \lambda_{j(k)} \stackrel{(s o)}{\longrightarrow} F \in \Omega$. This subnet is such that

$$
\begin{aligned}
\left\langle F(a), a^{*}\right\rangle \longleftarrow & \left\langle T \lambda_{j(k)}(a), a^{*}\right\rangle=\int_{\sigma_{\ell}}\left\langle g(a), a^{*}\right\rangle d \lambda_{j}(g) \\
& \longrightarrow \int_{\sigma^{\prime} \zeta}\left\langle g(a), a^{*}\right\rangle d \mu(g)=\left\langle T \mu(a), a^{*}\right\rangle,
\end{aligned}
$$

so that $T \mu=F$. Hence $T \mu$ is the only (so) limit point in $\Omega$ of $\left\{T \lambda_{j}\right\}$; since there exists at least one such limit point we conclude that $T \lambda_{j} \stackrel{(s o)}{\longrightarrow} T \mu$ (and in particular $T \mu \in \Omega$ ). Once we know that $T \mu_{j} \in \Omega$ for all $j \in J$ the same limit point argument applies to show that $T \mu_{j} \stackrel{(s o)}{\longrightarrow} T \mu$, as required for continuity. In particular, if $\left\{\mu_{j}: j \in J\right\}$ is a net in $N \cap \Sigma_{M\left(\sigma_{\ell}\right)}$ such that $\mu_{j} \stackrel{(\sigma)}{\longrightarrow} \mu \in M\left(G_{\ell}\right)$, then $0 \equiv T \mu_{j} \stackrel{(s o)}{\longrightarrow} T \mu$, so that $\mu \in N$. The Krein-Smulian theorem ([7], V.5.7) applies to show that $N$ is a $(\sigma)$ closed subspace in $M\left(G_{\ell}\right)$.

If $g_{0} \in G_{\ell}$ we have $T\left(\delta_{g_{0}}\right)=g_{0}$, so $T\left(\sum_{i=1}^{n} \alpha_{i} \delta_{g_{i}}\right)=\sum_{i=1}^{n} \alpha_{i} g_{i}$. If $F \in \Sigma_{A_{\ell}^{m}}$ there exists a net $\left\{E_{j}=\sum \alpha(j, g) g\right\}$ in co $\left[G_{\ell}\right]$ such that $E_{j} \stackrel{(s o)}{\longrightarrow} F$; thus, if we define $\lambda_{j}=\sum \alpha(j, g) \delta_{g}$ in $\Sigma_{M(G \zeta)}$, and if we: pick any $(\sigma)$ convergent subnet $\lambda_{j(k)} \stackrel{(\sigma)}{\longrightarrow} \mu$, we get $\mu \in \Sigma_{M(\theta \ell)}$ and

$$
F \stackrel{(s o)}{\longleftarrow} E_{j(k)}=T\left(\lambda_{j(k)}\right) \stackrel{(s o)}{\longrightarrow} T \mu
$$

because $T$ is continuous. Hence $F=T \mu$ and we have $T\left(\Sigma_{M\left(G_{\ell}\right)}\right)=$ $\Sigma_{A^{m}}$. Another way of viewing this fact is to say that, given any $F \in A_{\ell}^{m}$, there is some $\mu \in M\left(G_{\ell}\right)$ with $\|\mu\|=\|F\|$ and $T \mu=F$.

In the above representation scheme we actually have $T\left(L^{1}\left(G_{\ell}\right)\right) \subset A_{\ell}$ (inner multipliers). To prove this, recall that $A_{\ell}$ is (trivially) a left ideal in $A_{\ell}^{m}$ and that $f \in \mathscr{A}\left(L^{1}\left(G_{\ell}\right)\right) \Rightarrow\left\{T f \circ g=T\left(f * \delta_{g}\right): g \in G_{\ell}\right\}$ lies within some finite dimensional subspace $X=X(f)$ in $A_{\ell}$. If $\left\{\lambda_{j}\right\} \subset \operatorname{co}\left[G_{\ell}\right]$ is chosen so $\lambda_{j} \stackrel{(s o)}{\longrightarrow} L_{a}$, then $T f \circ \lambda_{j} \stackrel{(s o)}{\longrightarrow} T f \circ L_{a}$; this means $T f \circ L_{a} \in X$ for all $a \in A$ and clearly $T f \circ L_{a} \in A_{\iota}$ too. If $\left\{e_{j}\right\}$ is the approximate identity in $A$ then $T f \circ L_{e_{j}} \stackrel{(s o)}{\longrightarrow} T f$, but $T f \circ L_{e_{j}} \in X \subset A_{\ell}$ and the space $X$ is (so) closed since it is finite dimensional. Hence $T f \in A_{\ell}$. If 
$f \in L^{1}\left(G_{\ell}\right)$ we can use the fact that $\|T\| \leqq 1$ and $\mathscr{A}\left(L^{1}\right)$ is norm dense in $L^{1}\left(G_{\ell}\right)$ to show that $T f \in A_{\ell}$. It is now easy to show $T\left(L^{1}\left(G_{\ell}\right)\right)$ is norm dense in $A_{\ell}$, so that $A_{\ell}=\overline{T\left(L^{1}\left(G_{\ell}\right)\right)}$ : if $\left\{e_{j}\right\}$ is the approximate identity in $L^{1}\left(G_{\ell}\right)$ we have $e_{j} \stackrel{(\sigma)}{\longrightarrow} \delta_{e}$ which $\Rightarrow F_{j}=T\left(e_{j}\right) \stackrel{(s o)}{\longrightarrow} I$ in $A_{\ell}^{m}$; thus $\left\|F_{j}(a)-a\right\|=\left\|T e_{j}(a)-a\right\| \rightarrow 0$ for $a \in A$. But $L_{T^{\prime} e_{j}(a)} \in T\left(L^{1}\left(G_{\ell}\right)\right)$, because if $\mu \in M\left(G_{\ell}\right)$ is chosen such that $T \mu=L_{a}$ we have $L_{T^{\prime} \jmath^{\prime}(a)}=$ $T e_{j} \circ T \mu=T\left(e_{j} * \mu\right)$. Clearly $\left\|L_{T_{e j}(a)}-L_{a}\right\|=\left\|T e_{j}(a)-a\right\| \rightarrow 0$, since $L: A \rightarrow A_{l}$ is an isometry.

Finally, if $\varphi: M\left(G_{\ell}\right) \rightarrow M\left(G_{\ell}\right) / N$ is the canonical homomorphism, consider the mappings displayed in Figure 1 (here $A=T \circ \varphi^{-1}$ is obviously an isomorphism from $M\left(G_{\ell}\right) / N$ onto $\left.A_{\ell}^{m}\right)$.

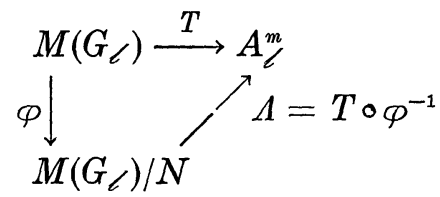

Figure 1

From the definition of quotient norm we see that, if $F \in A_{\ell}^{m}$ and if $\mu \in M\left(G_{\ell}\right)$ is chosen so that $\|\mu\|=\|F\|$ and $T \mu=F$, then

$$
\begin{aligned}
\|\mu\|=\|F\| & =\|T \mu\|=\inf \{\|T(\mu+n)\|: n \in N\} \\
& \leqq \inf \{\|\mu+n\|: n \in N\}=\|\varphi \mu\| \leqq\|\mu\| ;
\end{aligned}
$$

hence, $\Lambda$ is an isometric isomorphism. It is clear that $\Lambda$ maps $\left.\overline{\varphi\left(L^{1}\left(G_{\ell}\right)\right.}\right)$ isometrically and isomorphically onto $A_{\ell}$, which in turn is isometric and isomorphic to $A$.

In the above proof we have derived a considerable amount of information not mentioned explicitly in 2.1.4. In particular we can take $H=\left(G_{\ell},(s o)\right)$ there. By applying the structure theory of semisimple dual algebras, we can obtain the following representation.

THEOREM 2.1.5. Let $A$ be a semi-simple dual Banach algebra with central minimal approximate identity and assume that $A$ satisfies axiom $\left(C A_{\ell}\right)$. Then all the conclusions of 2.1 .4 are valid.

Proof. The discussion of Kaplansky [10] applies, and the existence of a central minimal approximate identity insures that all minimal closed two-sided ideals in $A$ are finite dimensional (see A.2.1). The linear span of these ideals is norm dense in $A$ (see [10], Theorem 5) and any finite dimensional two-sided ideal lies in $\mathscr{A}(A)$, so that $\mathscr{A}(A)$ is norm dense in $A$ and in particular axiom $\left(A I_{\ell}\right)$ is satisfied.

In later discussions concerning algebras with a nonzero multiplicative linear functional it will be essential to know that, if there exists 
an internal direct product factorization $G_{\ell}=S I \times \Gamma_{\ell}$, where $\Gamma_{\ell}$ is some (so) closed normal subgroup of $G_{\ell}$, we can reproduce the above representation theorems using $\Gamma_{\ell}$ instead of $G_{\ell}$.

THEOREM 2.1.6. Let $A$ be a Banach algebra with minimal twosided approximate identity and assume that $A$ satisfies axioms $\left(A I_{\ell}\right)$ and $\left(C A_{\ell}\right)$. Suppose there is an internal direct product factorization of $G_{\ell}=S I \times \Gamma_{\ell}$ where $S I=\{\alpha I:|\alpha|=1\}$ and $\Gamma_{\ell}$ is some (so) closed normal subgroup of $G_{\ell}$. Then there exists a $(\sigma)$ closed two-sided ideal $N$ in $M\left(\Gamma_{\ell}\right)$ such that $A_{\ell}^{m}$ is isometric and isomorphic to $M\left(\Gamma_{\ell}\right) / N$ and in this realization of $A_{\ell}^{m}$ we have $A_{\ell}$ identified with the norm closed subalgebra $\overline{\varphi\left(L^{1}\left(\Gamma_{\ell}\right)\right)}$, where $\varphi: M\left(\Gamma_{\ell}\right) \rightarrow M\left(\Gamma_{\ell}\right) / N$ is the canonical homomorphism and $M\left(\Gamma_{\ell}\right) / N$ is given the quotient norm.

Proof. If we define $T: M\left(\Gamma_{\ell}\right) \rightarrow A_{\ell}^{m}$ such that

$$
\left\langle T \mu(a), a^{*}\right\rangle=\int_{I_{\ell}}\left\langle\gamma(a), a^{*}\right\rangle d \mu(\gamma)
$$

for $a \in A, a^{*} \in A^{*}$, and $\mu \in M\left(\Gamma_{\ell}\right)$, the details of the proof are practically the same as in 2.1.4.

REMARK. If a Banach algebra $A$ has a nonzero multiplicative functional, we are guaranteed that the sort of internal direct product factorization discussed in 2.1.6 exists. Furthermore, all such factorizations of $G_{\ell}$ are essentially the same ( $\Gamma_{\ell}$ is unique up to a topological isomorphism). These facts are discussed in A.3.3. The significance of such factorizations when $A$ is a group algebra can be seen from 1.3.3.

2.2. Characterization of QCG algebras. We digress to study QCG algebras and prove the characterization stated in the summary. It will be convenient to call an algebra $A$ an $S M A$ algebra (sum of matrix algebras) if it is an internal algebraic direct sum of its nonzero finite dimensional minimal two-sided ideals. We will deal with algebras over the complex numbers, so each of these ideals is isomorphic to the full algebra $M(m, C)$ of complex $m \times m$ matrices for some $m=1,2 \cdots$; whenever we speak of a matrix algebra in the remaining discussion, we will mean an algebra of the form $M(m, C)$ for some $m \geqq 1$. An idempotent $e \neq 0$ in an algebra $A$ is a minimal idempotent if $e A e$ is a division ring, and is a minimal central idempotent if $e$ is central in $A$ and if $e e^{\prime}=e^{\prime} e=e$ for any central idempotent $e^{\prime} \in A$ such that $e e^{\prime} \neq 0$. It is well known that, if $A$ contains no proper ideal $I$ with $I^{2}=(0)$, then $A e(e A)$ are minimal left (right) ideals if $e$ is a minimal 
idempotent, and the two-sided ideal $e A=A e$ is minimal if $e$ is a minimal central idempotent in $A$ (see Rickart [14], p. 45-46).

THEOREM 2.2.1. Let $A$ be a QCG algebra represented as $A=$ $\overline{\varphi\left(L^{1}(H)\right)}$, where $H$ is a compact group, $N$ is a $(\sigma)$ closed two-sided ideal in $M(H)$, and $\varphi: M(H) \rightarrow M(H) / N$ is the canonical homomorphism. Then $A$ has the following general properties.

(1) $A$ is a semi-simple dual Banach algebra.

(2) If $\left\{E_{\alpha}: \alpha \in Q\right\}$ is the set of minimal closed two-sided ideals in $A$, these are all finite dimensional and their sum (obviously an $S M A$ algebra) is a norm dense two-sided ideal in A. Axioms $\left(A I_{\ell}\right)$ and $\left(A I_{r}\right)$ are satisfied.

(3) The sets of almost invariant elements coincide so that $\mathscr{A}_{\ell}(A)=\mathscr{A}_{r}(A)=\mathscr{A}(A)$, and if $\mathscr{A}_{0}\left(L^{1}\right)=\left\{f \in \mathscr{A}\left(L^{1}(H)\right): f * N=\right.$ $N * f=(0)\}$, then $\varphi$ is an isomorphism of $\mathscr{L}_{0}\left(L^{1}\right)$ onto $\mathscr{A}(A)$.

(4) A has a central minimal approximate identity.

The translations and multipliers of $A$ have the following properties.

(5) $A$ is a two-sided ideal in $M(H) / N$ and $A_{l}^{m}$ is isometric and isomorphic with $M(H) / N$ under the map $L: x \rightarrow L_{x}$ for $x \in M(H) / N$. Similarly $A_{r}^{m}$ is isometric and linearly anti-isomorphic with $M(H) / N$ under the right multiplication map $R: x \rightarrow R_{x}$ for $x \in M(H) / N$.

(6) In this realization of $A_{\ell}^{m}$ we have $G_{\ell}(A)=\varphi\left(\mathscr{E}_{M(H)}\right)=$ $\mathscr{E}_{M(H) / N}$. Similarly $G_{r}(A)=\varphi\left(\mathscr{E}_{M(H)}\right)=\mathscr{E}_{M(H) / N}$ in this realization of $A_{r}^{m}$.

(7) The convex span of $G_{\ell}(A)$ is (so) dense in $\Sigma_{A_{\ell}^{m}}$, so axiom $\left(C A_{\ell}\right)$ is satisfied by $A$. Similarly axiom $\left(C A_{r}\right)$ is satisfied.

(8) The norm closed left (right) ideals in $A$ are precisely the closed $G_{\ell}(A)$ invariant $\left(G_{r}(A)\right.$ invariant $)$ linear subspaces of $A$, so axioms $\left(W_{\ell}\right)$ and $\left(W_{r}\right)$ are satisfied.

(9) The representation map $L \circ \varphi:(M(H),(\sigma)) \rightarrow\left(A_{\ell}^{m},(s o)\right)$ is continuous on norm bounded sets, and similarly for $R \circ \varphi:(M(H),(\sigma)) \rightarrow$ $\left(A_{r}^{m},(s o)\right)$.

Proof. Since $M(H) * L^{1}(H) * M(H) \subset L^{1}(H)$, which implies $M(H) / N$. $\varphi\left(L^{1}(H)\right) \cdot M(H) / N \subset \varphi\left(L^{1}(H)\right)$, we conclude that $A=\overline{\varphi\left(L^{1}(H)\right)}$ is a two-sided ideal in $M(H) / N$ (multiplication is jointly norm continuous). Thus $L_{x}$ and $R_{x}$ are well defined operators (in $A_{\ell}^{m}$ and $A_{r}^{m}$ respectively) for $x \in M(H) / N$. If $\left\{f_{j}: j \in J\right\}$ is a central minimal approximate identity in $L^{1}(H)$ (see A.1.3) then $\varphi\left(f_{j}\right) \in A, \lim \sup \left\{\left\|\varphi f_{j}\right\|: j \in J\right\} \leqq 1$, and the elements $\varphi f_{j}$ are central in $A$. We have $\left\|f_{j} * f-f\right\| \rightarrow 0$ for $f \in L^{1}(H)$, so that $\left\|\varphi f_{j} \cdot \varphi f-\varphi f\right\| \rightarrow 0$ for $f \in L^{1}(H)$ and because $\varphi\left(L^{1}(H)\right)$ is norm dense in $A$ it follows that $\left\{\varphi f_{j}\right\}$ is an approximate identity in $A$. In particular we must have $\lim \left\{\left\|\varphi f_{j}\right\|: j \in J\right\}=1$, and part (4) 
is proved.

To prove the left-handed form of (9) we consider the $(s o)_{/}$topology gotten by letting $M(H)$ act on $L^{1}(H)$ by left convolution. In the appendix we show that $(s o)_{\ell}$ coincides with the $(\sigma)$ topology on norm bounded sets in $M(H)$ (see A.1.1 for a self contained exposition); thus if $\mu_{j} \stackrel{(\sigma)}{\longrightarrow} \mu$ is a norm bounded net, it follows that

$$
\left\|\varphi\left(\mu_{j}\right) \varphi f-\varphi(\mu) \varphi f\right\|=\left\|\varphi\left(\mu_{j} * f\right)-\varphi(\mu * f)\right\| \leqq\left\|\mu_{j} * f-\mu * f\right\| \rightarrow 0
$$

for $f \in L^{1}(H)$. Density of $\varphi\left(L^{1}(H)\right)$ in $A$ insures that $\left\|\varphi\left(\mu_{j}\right) a-\varphi(\mu) a\right\| \rightarrow$ 0 for $a \in A$, as required. A similar argument gives the right-handed version of (9).

To prove (5) we first observe that $\varphi\left(\Sigma_{M(H)}\right)=\Sigma_{M(H) / N}$; this simple fact is verified in A.1.2 as part of a general discussion of measure algebras on compact groups. Now consider the left multiplications $\left\{L_{x}: x \in M(H) / N\right\}$ on $A$; we will prove that $L: M(H) / N \rightarrow A^{m}$ is an isometric isomorphism. It is clear that $L$ is an isomorphism if we can show that for $x \neq 0$ in $M(H) / N$, there exists $b \in A$ such that $x b \neq 0$. Consider $\left\{e_{j}=\varphi f_{j}\right\}$ where $\left\{f_{j}: j \in J\right\}$ is the approximate identity in $L^{1}(H)$; if $\varphi \mu=x$ and if $x e_{j}=0$ for all $j \in J$, then $\mu * f_{j} \in N$ and $\mu * f_{j} \stackrel{(\sigma)}{\longrightarrow} \mu$, so that $\mu \in N$ and $\varphi \mu=x=0$. Next we show $L$ is isometric. If $\mu \in M(H)$ then

$$
\begin{aligned}
\|\mu\| & \geqq\|\varphi \mu\| \geqq\left\|L_{\varphi_{\mu}}\right\| \\
& =\sup \left\{\frac{\|\varphi(\mu) \cdot a\|}{\|a\|}: a \in A, a \neq 0\right\} \\
& \geqq \sup \left\{\frac{\|\varphi(\mu) \cdot a\|}{\|a\|}: a \in \varphi\left(L^{1}(H)\right), a \neq 0\right\} \\
& =\sup \left\{\frac{\|\varphi(\mu * f)\|}{\|\varphi f\|}: f \in L^{1}(H) \backslash N\right\} \\
& \geqq \lim \sup \left\{\frac{\left\|\varphi\left(\mu * f_{j}\right)\right\|}{\left\|\varphi f_{j}\right\|}: j \in J\right\} \\
& =\lim \sup \left\{\left\|\varphi\left(\mu * f_{j}\right)\right\|: j \in J\right\}
\end{aligned}
$$

since $\left\|e_{j}\right\|=\left\|\varphi f_{j}\right\| \rightarrow 1$. Now observe that

$$
\left\|\varphi\left(\mu * f_{j}\right)\right\|=\inf \left\{\left\|\mu * f_{j}+n\right\|: n \in N\right\}=\left\|\mu * f_{j}+n_{j}\right\|
$$

for some $n_{j} \in N$, since we have $\varphi\left(\Sigma_{M(H)}\right)=\Sigma_{M(B) / N}$. Furthermore,

$$
\left|\left\|n_{j}\right\|-\left\|\mu * f_{j}\right\|\right| \leqq\left\|\mu * f_{j}+n_{j}\right\|=\left\|\varphi\left(\mu * f_{j}\right)\right\| \leqq\|\mu\|
$$

for $j \in J$, so that $\left\|n_{j}\right\| \leqq 2\|\mu\|$. Hence there is a $(\sigma)$ convergent subnet $n_{j(k)} \stackrel{(\sigma)}{\longrightarrow} n_{0} \in N$. Now $f_{j} \stackrel{(\sigma)}{\longrightarrow} \delta_{e}$ in $M(H)$, so we have 


$$
n_{j(k)}+\mu * f_{j(k)} \stackrel{(\sigma)}{\longrightarrow} n_{0}+\mu
$$

(multiplication is separately $(\sigma)$ continuous in $(M(H))$. These remarks show that

$$
\begin{aligned}
\|\varphi \mu\| & \leqq\left\|\mu+n_{0}\right\| \\
& \leqq \lim \sup \left\{\left\|\mu * f_{j(k)}+n_{j(k)}\right\|: k \in K\right\} \\
& \leqq \lim \sup \left\{\left\|\mu * f_{j}+n_{j}\right\|: j \in J\right\} \\
& =\lim \sup \left\{\left\|\varphi\left(\mu * f_{j}\right)\right\|: j \in J\right\} .
\end{aligned}
$$

Combining this with $(*)$ we get $\|\varphi \mu\|=\left\|L_{\varphi \mu}\right\|$ for all $\mu \in M(H)$, as required. Finally, we must show $L$ is onto (so $A_{\ell}^{m}=\left\{L_{x}: x \in M(H) / N\right\}$ ). If $F \in A_{b}^{m}, a \in A$, and if $e_{j}=\varphi f_{j}$, then we have $\left\|\varphi f_{j} \cdot a-a\right\| \rightarrow 0$, which $\Rightarrow\left\|F\left(e_{j} a\right)-F(a)\right\|=\left\|F\left(e_{j}\right) a-F(a)\right\| \rightarrow 0$; this means that $L_{F(e j)} \stackrel{(s o)}{\longrightarrow} F$. But there exist $\lambda_{j} \in M(H)$ with $\left\|\lambda_{j}\right\|=\left\|F\left(e_{j}\right)\right\|$ and $\varphi\left(\lambda_{j}\right)=F\left(e_{j}\right)$, and there is a convergent subnet $\lambda_{j(k)} \stackrel{(\sigma)}{\longrightarrow} \mu \in M(H)$ since $\left\|\lambda_{j}\right\| \leqq\|F\|<\infty$. From (9) it follows that $L_{\varphi \lambda_{j(k)}} \stackrel{(s o)}{\longrightarrow} L_{\varphi \mu}$, which $\Rightarrow\left\|F\left(e_{j(k)}\right) \cdot a-\varphi \mu \cdot a\right\| \rightarrow 0$ for $a \in A$, while $\left\|F\left(e_{j}\right) \cdot a-F(a)\right\| \rightarrow 0$; thus $L_{\varphi \mu}=F$, proving (5). The modifications needed to identify $A_{r}^{m}$ with $M(H) / N$ are trivial.

In the above realization of $A_{\ell}^{m}$ we will show that

$$
\mathscr{E}_{M(H) / N} \subset \varphi\left(\mathscr{E}_{M(H)}\right) \subset G_{\ell}(A) \subset \mathscr{E}_{M(H) / N},
$$

to prove (6). We have $\varphi\left(\mathscr{E}_{M(H)}\right) \subset G_{\ell}$ because $\left\|L_{\varphi\left(\delta_{h}\right)}\right\|=\left\|\varphi\left(\delta_{h}\right)\right\| \leqq 1$, $\left\|L_{\varphi\left(\delta_{h}-1\right)}\right\|=\left\|\varphi\left(\delta_{h-1}\right)\right\| \leqq 1$, and if $a \in A$ we have $L_{\varphi\left(\delta_{h}\right)} L_{\varphi\left(\delta_{h-1}\right)}(a)=$ $L_{\varphi\left(\delta_{h-1}\right)} L_{\varphi\left(\delta_{h}\right)}(a)=a$ so $L_{\varphi\left(\delta_{h}-1\right)}=\left(L_{\varphi\left(\delta_{h}\right)}\right)^{-1}$; hence these are isometric onto multipliers of $A$. Next observe that $\mathscr{E}_{M(H) / N} \subset \varphi\left(\mathscr{E}_{M(H)}\right)$; in fact if $x \in \mathscr{E}_{M(H) / N}$, let us select some $\mu \in M(H)$ with $\varphi \mu=x$ and $\|\mu\|=$ $\|x\|=1$. Then if $0<\alpha<1$ and measures $\mu_{1}, \mu_{2}$ with $\left\|\mu_{i}\right\| \leqq 1$ are such that $\mu=\alpha \mu_{1}+(1-\alpha) \mu_{2}$, this $\Rightarrow x=\alpha \varphi\left(\mu_{1}\right)+(1-\alpha) \varphi\left(\mu_{2}\right)$, which $\Rightarrow \varphi \mu_{1}=\varphi \mu_{2}$. If $\operatorname{supp}(\mu)$ is not a single point, there exist measurable sets $A, B$ with $|\mu|(A) \neq 0,|\mu|(B) \neq 0, A \cup B=\operatorname{supp}(\mu)$, and $A \cap B=$ $\varnothing$. Define $\lambda_{A}=(\mu \mid A) /|\mu|(A)$ and $\lambda_{B}=(\mu \mid B) /|\mu|(B)$, we have $\mu=$ $|\mu|(A) \lambda_{A}+|\mu|(B) \lambda_{B}$ with $|\mu|(A)+|\mu|(B)=\|\mu\|=1$; hence $\varphi\left(\lambda_{A}\right)=$ $\varphi\left(\lambda_{B}\right)=x$. In particular, $\lambda_{A}(\mu)=(\mu \mid A) /|\mu|(A)$ is in $\mathfrak{S}_{M(H)} \cap \varphi^{-1}(x)$ if $\mu \in \mathfrak{S}_{M(H)} \cap \varphi^{-1}(x),\left(\mathfrak{S}_{M(H)}=\{\lambda \in M(H):\|\lambda\|=1\} \subset \Sigma_{M(H)}\right)$ and if $A$ is any measurable set in supp $(\mu)$ such that $|\mu|(A) \neq 0$.

Let $\left\{U_{j}: j \in J\right\}$ be a base of neighborhoods for some $h_{0} \in \operatorname{supp}(\mu)$, with $J$ partially ordered so that $j_{1}>j_{2} \Leftrightarrow U_{j_{1}} \subset U_{j_{2}}$. Define $A_{j}=$ $\operatorname{supp}(\mu) \cap U_{j}$; we have $|\mu|\left(A_{j}\right)>0$ for each $j \in J$. If $|\mu|\left(h_{0}\right) \neq 0$, then by taking $A=\left\{h_{0}\right\}$ above, we see that $\delta_{h_{0}} \in \mathfrak{S}_{M(H)} \cap \varphi^{-1}(x)$, so $x \in \varphi\left(\mathscr{E}_{\boldsymbol{M}(\boldsymbol{H})}\right)$, as desired. Otherwise we have $|\mu|\left(h_{0}\right)=0$ for all $h_{0} \in \operatorname{supp}(\mu)$ and all $\mu \in \mathfrak{S}_{(M) H} \cap \varphi^{-1}(x)$, so that these measures are all 
continuous on $H$. But this means that $|\mu|\left(A_{j}\right) \rightarrow 0$ since $\mu$ is a regular Borel measure. Now form the net $\left\{\lambda_{j}=\left(\mu \mid A_{j}\right) /|\mu|\left(A_{j}\right): j \in J\right\}$ in $\mathfrak{S}_{M(H)} \cap \varphi^{-1}(x)$. The latter set is $(\sigma)$ compact since $\varphi^{-1}(x)$ is $(\sigma)$ closed in $M(H)$ and $\widetilde{S}_{M(H)} \cap \varphi^{-1}(x)=\Sigma_{M(H)} \cap \varphi^{-1}(x)$; indeed, $\|x\|=1 \Rightarrow$ $\|\mu\| \geqq 1$ for any $\mu \in \varphi^{-1}(x)$. Thus there is a $(\sigma)$ convergent subnet with limit $\lambda_{0} \in \mathcal{S}_{M(H)} \cap \varphi^{-1}(x)$, and from the nature of $\operatorname{supp}\left(\lambda_{j}\right)$ it is clear that we must have supp $\left(\lambda_{0}\right)=\left\{h_{0}\right\}$, a contradiction. In discussing the translations of general Banach algebras in the appendix (see A.3.1) we show that translations are always extreme points in $\Sigma_{A^{m}}$; thus $G_{\ell}(A) \subset \mathscr{E}_{M(H) / N}$ here and the proof of (6) is complete. It is well known that $\operatorname{co}\left[\mathscr{E}_{M(H)}\right]$ is $(\sigma)$ dense in $\Sigma_{M(H)}$, and we have shown $L \circ \varphi\left(\Sigma_{M(H)}\right)=\Sigma_{\Lambda_{\ell}^{m}}$, thus $L \circ \varphi\left(\operatorname{co}\left[\mathscr{E}_{M(H)}\right]\right)=\operatorname{co}\left[G_{\ell}(A)\right]$ is (so) dense in $\Sigma_{A_{C}^{m}}$ from the continuity of $L \circ \varphi$. The righthanded situation is treated similarly to prove (7). For (8), notice that existence of a minimal approximate identity in $A$ implies that any closed left (right) ideal in $A$ is left (right) translation invariant. Conversely, translation invariance of a closed subspace $E$ implies $\lambda(E) \subset E$ for all $\lambda \in \operatorname{co}\left[G_{\ell}\right]$; but if $a \in \Sigma_{A}$ there is a net $\left\{\lambda_{j}\right\}$ in co $\left[G_{\ell}\right]$ such that $\lambda_{j} \stackrel{(s o)}{\longrightarrow} L_{a}$, and hence $L_{a}(E)=a \cdot E \subset E$.

We are ready to deal with (2) and (3), which prove axioms $\left(A I_{\ell}\right)$ and $\left(A I_{r}\right)$ for $A$. Let $\mathscr{J}=\left\{f_{\beta}: \beta \in J\right\}$ be the minimal central idempotents in $L^{1}(H)$ and set $\mathscr{A}=\mathscr{A}\left(L^{1}(H)\right)$. Then the minimal two-sided of $L^{1}(H)$ are finite dimensional, are precisely $\left\{F_{\beta}=L^{1} * f_{\beta}: f_{\beta} \in \mathscr{I}\right\}$, and we have $\mathscr{A}=\sum \oplus\left\{F_{\beta}: f_{\beta} \in \mathscr{F}\right\}$ (internal direct sum of ideals). Let

$$
\mathscr{J}^{\prime}=\left\{f_{\beta} \in \mathscr{J}: f_{\beta} * N=N * f_{\beta}=(0)\right\}, \mathscr{I}^{\prime \prime}=\left\{f_{\beta} \in \mathscr{J}: f_{\beta} * L^{1} \subset N\right\}
$$

and form the internal direct sums

$$
E^{\prime}=\sum \oplus\left\{L^{1} * f_{\beta}: f_{\beta} \in \mathscr{J}^{\prime}\right\}, E^{\prime \prime}=\sum \oplus\left\{L^{1} * f_{\beta}: f_{\beta} \in \mathscr{J}^{\prime \prime}\right\} ;
$$

these are two-sided ideals and $\mathscr{A}=E^{\prime} \oplus E^{\prime \prime}$. Notice that minimality of $F_{\beta}$ as a two-sided ideal implies that either $F_{\beta} \cap N=(0)$ or $F_{\beta} \subset N$. The homomorphism $\varphi$ is faithful in $E^{\prime}$ and annihilates $E^{\prime \prime}$, so $\varphi\left(E^{\prime}\right)=$ $\varphi\left(\mathscr{A}\left(L^{1}\right)\right)$ is norm dense on $A$. We assert that $\mathscr{A}_{0}=\varphi\left(E^{\prime}\right)$ is precisely $\mathscr{A}(A)$. The density of $\varphi\left(L^{1}(H)\right)$ in $A$ insures that $e_{\beta}=\varphi\left(f_{\beta}\right)$ is a nonzero central idempotent in $A$ for each $f_{\beta} \in \mathscr{I}^{\prime}$; furthermore, if $E_{\beta}=\varphi\left(F_{\beta}\right)$, where $F_{\beta}=L^{1} * f_{\beta}$ for some $f_{\beta} \in \mathscr{J}^{\prime}$, then $E_{\beta}=\varphi\left(F_{\beta}\right)=$ $\varphi\left(L^{1}(H)\right) \cdot \varphi f_{\beta}=A e_{\beta}=e_{\beta} A$ (the last steps, because $E_{\beta}$ is finite dimensional) and $E_{\beta}$ is a nonzero two-sided ideal. It is actually minimal because $E_{\beta}$ is isomorphic to the (simple) complex matrix algebra $F_{\beta} \subset L^{1}(H)$. It follows that the idempotents $\mathscr{J}=\left\{e_{\beta}=\varphi\left(f_{\beta}\right): f_{\beta} \in \mathscr{J}^{\prime}\right\}$ are minimal central idempotents in $A$ and it is easy to see that there are no other such idempotents in $A$ because $\mathscr{A}_{0}=\sum \oplus\left\{E_{\beta}: f_{\beta} \in \mathscr{I}^{\prime}\right\}$ 
is norm dense in $A$.

It is obvious from finite dimensionality of each two-sided ideal $E_{\beta}$ (and existence of an approximate identity in $A$ ) that $\mathscr{A}_{0} \subset \mathscr{A}(A)$, since closed two-sided ideals of $A$ are $G_{\ell}(A)$ and $G_{r}(A)$ invariant. On the other hand, if $a \in \mathscr{A}(A)$ then $E_{a}=1$.s. $\left[G_{\ell}\left(G_{r}(a)\right)\right]=1$.s. $\left[G_{r}\left(G_{\ell}(a)\right)\right]$ is a left and right translation invariant finite dimensional subspace, which means $E_{a}$ is a two-sided ideal (see (8)). However, if $M$ is any finite dimensional two-sided ideal, then $M \cap E_{\beta}=M e_{\beta}=e_{\beta} M$ and this can be nonzero for only finitely many of the $E_{\beta}$ (recall $E_{\alpha} E_{\beta}=E_{\beta} E_{\alpha}=(0)$ unless $\alpha=\beta)$. Let $E_{1}=\sum \oplus\left\{E_{\beta} \cap E_{a}: e_{\beta} \in \mathscr{J}\right\}$. Then this sum is finite and $E_{1}$ is a two-sided ideal of $A$ lying within $E_{a}$; if $E_{1}=E_{a}$ then clearly $E_{a} \subset \mathscr{A}_{0}$ and we conclude $E_{a} \subset \mathscr{A}_{0}$ for each $a \in \mathscr{A}(A)$, which means that $\mathscr{A}(A) \subset \mathscr{A}_{0}$. But if $E_{1} \neq E_{a}$ there exists some nonzero $x \in E_{a}$ such that $x e_{\beta}=0$ for all $e_{\beta} \in \mathscr{J}$, so that $x \cdot \mathscr{A}_{0}=(0)$; however, norm density of $\mathscr{A}_{0}$ in $A$ gives us $x \cdot A=(0)$ which contradicts the existence of an approximate identity in $A$. Notice that, in proving (2) and (3), we have also demonstrated that $\mathscr{A}(A)$ is an SMA algebra norm dense in $A$.

Only (1) is left to prove. If $x \in A$ then the norm closures of $x A$ and $A x$ both contain $x$ since there is a two-sided approximate identity, and $A$ contains a norm dense subalgebra which is a dual algebra (all SMA algebras, such as $\mathscr{A}(A)$, are dual: see [10], Theorem 6, p. 693). But these conditions insure that $A$ is a dual algebra (see [10], Theorem 7, p. 694 for this). Since $A$ is a Banach algebra its radical is a closed two-sided ideal, so its radical is either zero or contains one of the minimal two-sided ideals in $E_{\beta}$ (due to density of $\mathscr{A}_{0}$ in $A$ ); the latter situation is impossible, since it would imply that there is a nonzero idempotent in the radical (see Rickart [14], p. 56).

Parts (2), (3), (4), and (7) of 2.2.1 give the converse of 2.1.4. The following characterization of QCG algebras is immediate.

THEOREM 2.2.2. A Banach algebra $A$ with minimal two-sided approximate identity is a $Q C G$ algebra $\Leftrightarrow A$ satisfies axioms $\left(A I_{\ell}\right)$ and $\left(C A_{\ell}\right)$.

THEOREM 2.2.3. A Banach algebra $A$ is a QCG algebra $\Leftrightarrow$ it is a semi-simple dual algebra, with central minimal approximate identity, which satisfies axiom $\left(C A_{\ell}\right)$.

Proof. The verification $(\Leftrightarrow)$ is in 2.2.1, while $(\Leftrightarrow)$ is given in 2.1.7.

The following observations are also clear from 2.2.1. 
CoRollary 2.2.4. If $A$ is a $Q C G$ algebra then $A$ can be represented as $A=\overline{\varphi\left(L^{1}\left(G_{\ell}\right)\right)}$ in $M\left(G_{\ell}\right) / N$, where $G_{\ell}(A)$ is taken with the (so) topology, $N$ is some $(\sigma)$ closed two-sided ideal in $M\left(G_{\ell}\right)$, and $\varphi: M\left(G_{\ell}\right) \rightarrow M\left(G_{\ell}\right) / N$ is the canonical homomorphism.

REMARK. If $H$ is a compact group and $N$ is a $(\sigma)$ closed twosided ideal in $M(H)$, then the norm closed subalgebra $A=\overline{\varphi\left(L^{1}(H)\right)}$ in $M(H) / N$, where $\varphi: M(H) \rightarrow M(H) / N$ is the canonical homomorphism, is a typical QCG algebra. Generally $N \not \subset L^{1}(H)$, so it is somewhat surprising that $\varphi\left(L^{1}(H)\right)$ itself is actually norm closed in $M(H) / N$, so that $A=\varphi\left(L^{1}(H)\right)$. In 2.1.4 this means that every QCG algebra can actually be represented as $A=\varphi\left(L^{1}\left(G_{\ell}\right)\right)$ in $M\left(G_{\ell}\right) / N$ for some $(\sigma)$ closed two-sided ideal $N \subset M\left(G_{\ell}\right)$; in the definition of QCG algebras (1.4.1) we can replace $\overline{\varphi\left(L^{1}(H)\right)}$ with $\varphi\left(L^{1}(H)\right)$.

Proposition 2.2.5. In the above scheme, the norm closure of $\varphi\left(\Sigma_{L^{1}(H)}\right)$ coincides with $\Sigma_{A}$ and if $\varepsilon>0$ then $\varphi\left((1+\varepsilon) \cdot \Sigma_{L^{1}(H)}\right) \supset \Sigma_{A}$. Thus $\varphi\left(L^{1}(H)\right)$ is norm closed in $M(H) / N$.

Proof. We have remarked in proving part (4) of 2.2.1, that $\left\{\varnothing e_{j}: j \in J\right\}$ is a minimal two-sided approximate identity in $A$ if $\left\{e_{j}\right\}$ is one in $L^{1}(H)$; we also showed that $\varphi\left(\Sigma_{M(H)}\right)=\Sigma_{M(H) / N}$, and clearly $\varphi\left(L^{1}(H)\right)$ is a norm dense subalgebra in $A$. Given $a \in A$ and $\varepsilon>0$ there exists $f \in L^{1}(H)$ such that $\|f\| \leqq\|a\|$ and $\|\varphi f-a\|<\varepsilon$; in fact, we can write $a=\varphi \mu$ for some $\mu \in M(H)$ with $\|\mu\|=\|a\|$ and we have $\varphi\left(e_{j} * \mu\right)=\varphi\left(e_{j}\right) \varphi(\mu)=\varphi\left(e_{j}\right) \cdot a \stackrel{\text { norm }}{\longrightarrow} a$, while $\left\|e_{j} * \mu\right\| \leqq\|\mu\|=$ $\|a\|$, so we can take $f=e_{j} * \mu$ for some index $j \in J$. Now for $\varepsilon>0$, $a \in A,\|a\|=1$ take $f_{1} \in L^{1}(H)$ such that $\left\|f_{1}\right\| \leqq 1$ and $\left\|\varphi f_{1}-a\right\|<\varepsilon / 2$. Then $\left(\varphi f_{1}-a\right) \in A$ and there exists $f_{2} \in L^{1}(H)$ such that $\left\|f_{2}\right\| \leqq$ $\left\|\varphi f_{1}-a\right\|<\varepsilon / 2$ and $\left\|\varphi f_{2}-\left(a-\varphi f_{1}\right)\right\|<\varepsilon / 4$. Continuing, we see that $\left(a-\sum_{i=1}^{n-1} \varphi f_{i}\right) \in A$ and there exists $f_{n} \in L^{1}(H)$ such that $\left\|f_{n}\right\| \leqq$ $\left\|a-\sum_{i=1}^{n-1} \varphi f_{i}\right\|<\varepsilon / 2^{n-1}$ and $\left\|\varphi f_{n}-\left(a-\sum_{i=1}^{n-1} \varphi f_{i}\right)\right\|<\varepsilon / 2^{n}$. Hence $f=\sum_{n=1}^{\infty} f_{n} \in L^{1}(H),\|f\|<1+\varepsilon$, and $\varphi f=\sum_{n=1}^{\infty} \varphi f_{n}=a$ is uniformly convergent in $A$.

2.3. QCG algebras which are not group algebras, As an example we construct a class of commutative, semi-simple QCG algebras which have all properties of a group algebra listed in Section 1 and nevertheless fail to be group algebras.

EXAMPLE 1. Noncommutative QCG algebras which are not group algebras. If $H$ is a finite, noncommutative group and $N \subset M(H)$ is any proper two-sided ideal which includes all one dimensional two-sided 
ideals, then $A=M(H) / N$ is a QCG algebra without multiplicative linear functionals and cannot be a group algebra.

EXAMPLE 2. Let $S$ be the circle group and $Z$ the additive integers (the character group of $S$ if we take $\langle n, s\rangle=s^{n}$ ), and define $H^{1}(S)=$ $\left\{\mu \in M(S): \mu^{\wedge}(n)=\int e^{-i n \theta} d \mu(\theta)=0\right.$ for all $\left.n>0\right\}$. Then $K=H^{1}(S)$ is clearly a $(\sigma)$-closed two-sided ideal in $M(S)$ which actually lies within $L^{1}(S)$ (see Hoffman [9], p. 47).

If $K$ is any $(\sigma)$ closed two-sided ideal in $M(S)$ such that $K \subset L^{1}(S)$, then $A=L^{1}(S) / K$ is a commutative QCG algebra. But $A$ is a group algebra only if $K$ has the following very special properties, and is certainly not a group algebra when $K$ is finite dimensional or $K=H^{1}(S)$.

Proposition 2.3.1. $A$ is a group algebra $\Leftrightarrow$ the cospectrum $\operatorname{cosp}(K)=\left\{n \in Z: \mu^{\wedge}(n)=0\right.$, all $\left.\mu \in K\right\}$ is a coset of some subgroup in $Z$.

Proof. Let $\mathscr{C l}_{A}$ be the maximal modular ideal space of $A$ and let $a \rightarrow\langle a, M\rangle$ be the multiplicative linear functional associated with $M \in \mathscr{C}_{A}$. These extend uniquely to multiplicative functionals $F \rightarrow$ $\langle F, M\rangle$ on $A^{m}$ if we require $\left\langle L_{a}, M\right\rangle=\langle a, M\rangle$ for $a \in A$ (see Birtel [1]). Let $G \subset A^{m}$ be the group of translations on $A$; it is clear that the functions $\alpha_{M}: g \rightarrow\langle g, M\rangle$ obtained by restricting these extended functionals to $G$ are (so) continuous multiplicative characters on $G$. Let $R=\left\{\alpha_{M}: M \in \mathscr{M}_{\Delta}\right\}$, a subset of $G^{\wedge}$ when $G$ is given the (so) topology.

In the following we fix one $M_{0} \in \mathscr{C}_{A}$ and make use of the internal direct product factorization $G=S I \times \Gamma_{0}$, where $S I=\{\alpha I:|\alpha|=1\}$ and $\Gamma_{0}=\left\{g \in G:\left\langle g, M_{0}\right\rangle=1\right\}$ (a (so) closed normal subgroup in $G$ ). Consider the functions in $R$ restricted to $\Gamma_{0}: R_{0}=\left\{\beta_{M}=\alpha_{M} \mid \Gamma_{0}: M \in \mathscr{C}_{A}\right\} \subset \Gamma_{0} \wedge$ and the subgroup $\Delta \subset \Gamma_{0}^{\wedge}$ generated by $R_{0}$. In $C\left(\Gamma_{0}\right)$ the linear span l.s. [U] is a subalgebra of functions on $\Gamma_{0}$ which contains all constants $\left(\beta_{M_{0}}=1\right)$, is closed under complex conjugation, and separates points (since $\left\langle\gamma_{1}, M\right\rangle=\left\langle\gamma_{2}, M\right\rangle$ all $M \in \mathscr{L}_{A} \Leftrightarrow \gamma_{1}=\gamma_{2}$ in $\Gamma_{0}$ ). If $R_{0}$ itself is a subgroup in $\Gamma_{0}^{\wedge}$, then $\Delta=R_{0}$ and $\alpha \in\left(\Gamma_{0}^{\wedge}\right) \backslash R_{0} \Rightarrow \int \alpha(\gamma) \overline{\beta(\gamma)} d \gamma=0$, all $\beta \in R_{0} \Rightarrow \int \alpha(\gamma) \overline{\Psi(\gamma)} d \gamma=0$, all $\Psi \in$ l.s. $[\Delta]=$ l.s. $\left[R_{0}\right]$ (sup norm dense in $\left.C\left(\Gamma_{0}\right)\right)$. This means $\alpha \equiv 0$, so $R_{0}=\Gamma_{0}^{\wedge}$ if $R_{0}$ is a subgroup.

We assert: if $R_{0}$ is defined as above, $A$ is a group algebra $\Leftrightarrow$ there exists an $M_{0} \in \mathscr{C}_{\Delta}$ such that $R_{0}$ is a subgroup in $\Gamma_{0}^{\wedge}$. The implication $\left(\Rightarrow\right.$ ) follows from the well known identification of $\mathscr{L}_{A}$ for group algebras 
(see also 1.3.3). Conversely we have $M_{0}$ such that $R_{0}=\Gamma_{0}^{\wedge}$; as in 2.1.6 we can construct a representation $T: M\left(\Gamma_{0}\right) \rightarrow A^{m}$ which realizes $A^{m} \sim M\left(\Gamma_{0}\right) / N$ and $A \sim L^{1}\left(\Gamma_{0}\right) / N$. But

$$
N=\operatorname{Ker}(T)=\left\{\mu \in M\left(\Gamma_{0}\right): T \mu(a)=\int_{\Gamma_{0}} \gamma(a) d \mu(\gamma)=0, \text { all } a \in A\right\},
$$

so $\mu \in N \Rightarrow$

$$
\begin{aligned}
0 & =\langle T \mu(a), M\rangle \\
& =\int_{\Gamma_{0}}\langle\gamma(a), M\rangle d \mu(\gamma) \\
& =\langle a, M\rangle \int_{\Gamma_{0}}\langle\gamma, M\rangle d \mu(\gamma) \\
& =\langle a, M\rangle \mu^{\wedge}\left(\overline{\beta_{M}}\right)
\end{aligned}
$$

for $M \in \mathscr{C}_{A}, a \in A$; i.e., $\mu^{\wedge}(\bar{\beta})=0$ for $\beta \in R_{0}=\Gamma_{0}^{\wedge}$, so $\mu=0$. Then $N=(0)$ and $A \sim L^{1}\left(\Gamma_{0}\right)$.

Let $\varphi: L^{1}(S) \rightarrow A=L^{1}(S) / K$ be the quotient map. The multiplicative functional $a \rightarrow\langle a, M\rangle$ induces a multiplicative functional $f \rightarrow\langle\varphi f, M\rangle$ on $L^{1}(S)$, so there is a unique $n \in \operatorname{cosp}(K)$ such that $\langle\varphi f, M\rangle=f^{\wedge}(n)$; conversely if $n \in \operatorname{cosp}(K)$ then $f \rightarrow f^{\wedge}(n)$ annihilates $K$ and induces a nonzero multiplicative functional $a \rightarrow\langle a, M(n)\rangle$ for some $M(n) \in \mathscr{I}_{A}$. Clearly this correspondence $n \rightarrow M(n)$ maps $\operatorname{cosp}(K)$ biuniquely onto $\mathscr{M}_{4}$.

Let $\widetilde{\varphi}: M(S) \rightarrow M(S) / K$ be the quotient map and define $L \circ \widetilde{\varphi}(\mu) \in A^{m}$ so $L \circ \widetilde{\varphi}(\mu): a \rightarrow \widetilde{\varphi}(\mu) \cdot a$. Then $L \circ \widetilde{\varphi}: M(S) \rightarrow A^{m}$ and $L \circ \widetilde{\varphi}: \mathscr{E}_{M(S)} \rightarrow G$ are onto homomorphisms (see 2.2.1). For $n \in \operatorname{cosp}(K), M(n) \in \mathscr{C}_{A}$ gives a functional $F \rightarrow\langle F, M(n)\rangle$ on $A^{m}$ such that $\left\langle L_{a}, M(n)\right\rangle=\langle a, M(n)\rangle$; it is evident that

$$
\langle L \circ \widetilde{\varphi}(f), M(n)\rangle=\langle\varphi(f), M(n)\rangle=f^{\wedge}(n) \quad \text { all } f \in L^{1}(S) .
$$

One can easily verify that we have:

$$
\langle L \circ \tilde{\varphi}(\mu), M(n)\rangle=\mu^{\wedge}(n)
$$

for $n \in \operatorname{cosp}(K), \mu \in M(S)$. The characters $\alpha_{M} \in R$ induced on $G$ by the $M \in \mathscr{M}_{A}$ are thus computed via the formula

$$
\left\langle L \circ \widetilde{\varphi}\left(\alpha \delta_{z}\right), M(n)\right\rangle=\alpha \delta_{z}^{\wedge}(n)=\alpha \overline{\langle n, z\rangle}=\alpha z^{-n},
$$

for $\alpha, z \in S$.

If $M_{0}=M(p) \in \mathscr{M}_{\boldsymbol{A}}$ is fixed $(p \in \operatorname{cosp}(K))$, it is clear from these computations that $\Gamma_{0}=\left\{g \in G:\left\langle g, M_{0}\right\rangle=1\right\}=L \circ \widetilde{\varphi}\left\{z^{p} \delta_{z}: z \in S\right\}$ and the characters $\beta_{M} \in R_{0} \subset \Gamma_{0}^{\wedge}$ are given by

$$
\beta_{M(n)}\left(L \circ \widetilde{\varphi}\left(z^{p} \delta_{z}\right)\right)=\left\langle L \circ \widetilde{\varphi}\left(z^{p} \delta_{z}\right), M(n)\right\rangle=z^{p-n}
$$


for $n \in \operatorname{cosp}(K), z \in S$. We have seen $A$ is a group algebra $\Leftrightarrow$ there exists an $M_{0} \in \mathscr{M}_{A}$ for which the set of characters $R_{0} \subset \Gamma_{0}^{\wedge}$ is a subgroup. It is easy to verify using the preceding computations, that $R_{0}$ is a subgroup for $M_{0}=M(p), p \in \operatorname{cosp}(K) \Leftrightarrow\{n-p: n \in \operatorname{cosp}(K)\}$ is a subgroup in $Z$. Obviously there exists $p \in \operatorname{cosp}(K)$ for which this happens $\Leftrightarrow \operatorname{cosp}(K)$ is a coset of a subgroup in $Z$.

\section{Characterization of QCG algebras which are group algebras.}

3.1. The regular representations of $G_{\ell}(A)$. The representation theory of a compact group $H$ is assumed. Our notation conforms to Chevalley [6]. We consider the class $R(H)$ of continuous representations $(V, E)$ on finite dimensional complex vector spaces $E$ (continuous means $h \rightarrow\left\langle V_{h}(x), x^{*}\right\rangle$ is continuous for $\left.x \in E, x^{*} \in E^{*}\right)$. Given $(V, E),(W, F) \in R(H)$ we are interested in the related representations $(V \oplus W, E \oplus F),(V \otimes W, L(E, F))$ where $L(E, F)$ is the space of linear mappings, and $\left(V^{*}, E^{*}\right)$. If representations $(V, E)$ and $(W, F)$ in $R(H)$ are equivalent we write $(V, E) \sim(W, F)$.

REMARK. Recall that $(V, E),\left(W, F^{\prime}\right) \in R(H)$ are equivalent $\Leftrightarrow$ the trace functions $\operatorname{tr}_{(V, E)}(h)=\operatorname{tr}_{(W, F)}(h)$ on $H$; we also have $(1) \operatorname{tr}_{V \oplus W}(h)=$ $\operatorname{tr}_{V}(h)+\operatorname{tr}_{W}(h), \quad(2) \operatorname{tr}_{V \otimes W}(h)=\operatorname{tr}_{V}(h) \cdot \operatorname{tr}_{W}(h)$, and (3) $\operatorname{tr}_{V^{*}}(h)=\overline{\operatorname{tr}_{V}(h)}$ on $H$.

If $A$ is a normed algebra we consider the class $R(A)$ of finite dimensional representations $(V, E)$ which are bounded $\left(\sup \left\{\left|\left\langle V_{a}(x), x^{*}\right\rangle\right|\right.\right.$ : $\|a\| \leqq 1\}<\infty$ for any $\left.x \in E, x^{*} \in E^{*}\right)$ and have dense range, so that $\left\{V_{a}(E): a \in A\right\}=E$.

Let $A$ be a QCG algebra. Consider the representation $(V, E)$ of $G_{\ell}(A)$ obtained by letting $G_{\zeta}$ act on a finite dimensional left ideal $E$ in $A$ (explicitly, $V_{g}: x \rightarrow g(x)$ for $x \in E$ ); we denote such representations as $(V, E)=\left(G_{\ell} \mid E\right)$. Clearly $\left(G_{\ell} \mid E\right)$ is (so) continuous on $G_{\ell}$. Define $R_{0}\left(G_{\ell}\right)$ to be the smallest set in $R\left(G_{\ell}\right)$ which is closed under direct sums and equivalences and contains all representations $\left(G_{\ell} \mid E\right)$ for finite dimensional left ideals $E$ in $A$. Our characterization of group algebras among QCG algebras is based on the algebraic structure of $R_{0}\left(G_{\ell}\right)$.

LEMMA 3.1.1. The set $R_{0}\left(G_{\ell}\right)$ contains every irreducible representation in $R\left(G_{\ell}\right)$ which belongs to one of its representations. Each irreducible representation in $R_{0}\left(G_{\ell}\right)$ is equivalent to $\left(G_{\ell} \mid E\right)$ for some (finite dimensional) minimal left ideal in $A$.

Proof. Every $(V, E) \in R\left(G_{\ell}\right)$ is semi-simple, since $G_{\ell}$ is compact; 
thus if $(V, E) \in R_{0}\left(G_{\ell}\right)$ there exist irreducible representations $\left\{\left(V^{i}, E_{i}\right)\right.$ : $i=1,2 \cdots n\}$ in $R\left(G_{\ell}\right)$ such that $(V, E) \sim\left(V^{1}, E_{1}\right) \oplus \cdots \oplus\left(V^{n}, E_{n}\right)$. It suffices to consider representations of the form $(V, E)=\left(G_{\ell} \mid E\right)$ for finite dimensional left ideals $E \subset A$, since any $(V, E) \in R_{0}\left(G_{\ell}\right)$ is equivalent to a direct sum of these. If $S: E \rightarrow E_{1} \oplus \cdots \oplus E_{n}$ is the isomorphism which gives the equivalence, then $I_{k}=S^{-1}\left(E_{k}\right)$ is a finite dimensional $G_{\zeta}$ invariant subspace of $A$ (indeed, $g\left(S^{-1}(y)\right)=V_{g}\left(S^{-1}(y)\right)=$ $S^{-1}\left(V_{g}^{k}(y)\right)$ for $\left.y \in E_{k}\right)$, so $I_{k}$ is a left ideal; furthermore $I_{j} \cap I_{k}=(0)$ if $j \neq k$ because $S$ is $1: 1$ and $I_{j} \cap I_{k}=S^{-1}\left(E_{j}\right) \cap S^{-1}\left(E_{j}\right)=S^{-1}\left(E_{j} \cap E_{k}\right)=$ (0). Clearly $S_{k}=S \mid I_{k}$ is an isomorphism of $I_{k}$ onto $E_{k}$ which gives the equivalence $\left(G_{\ell} \mid I_{k}\right) \sim\left(V^{k}, E_{k}\right)$. If $(V, E) \in R_{0}\left(G_{\ell}\right)$ is irreducible, then $(V, E) \sim\left(G_{\ell} \mid J\right)$ for some finite dimensional left ideal $J \subset A$; but $J$ must be minimal, because any proper subideal $J^{\prime} \subset J$ corresponds to a proper $G_{\ell}$ invariant subspace in $E$, contradicting irreducibility of $(V, E)$.

Corollary 3.1.2. We obtain $R_{0}\left(G_{\ell}\right)$ by taking all representations in $R\left(G_{\ell}\right)$ equivalent to direct sums $\left(G_{\ell} \mid E_{1}\right) \oplus \cdots \oplus\left(G_{\ell} \mid E_{n}\right)$ where the $I_{k}$ are minimal left ideals in $A$.

We can now state the characterization theorem; the rest of Section 3 is devoted to the proof of this result.

THEOREM 3.1.3. If $A$ is a QCG algebra then $A$ is the group algebra of some compact group $\Leftrightarrow$ there is a nonzero multiplicative linear functional on $A$ and $R_{0}\left(G_{\ell}\right)$ contains $V_{1} \otimes\left(V_{2}\right)^{*} \otimes V_{2}$ whenever $V_{1}, \cdots, V_{3}$ are in $R_{0}\left(G_{\ell}\right)$.

COROLLARY 3.1.4. If $A$ is a QCG algebra then $A$ is a group algebra $\Leftrightarrow$ there exists a nonzero multiplicative linear functional on $A$ and the collection of trace functions $T_{0}\left(G_{\ell}\right)=\left\{\operatorname{tr}_{(V, E)}:(V, E) \in R_{0}\left(G_{\ell}\right)\right\}$ is closed under formation of the pointwise products $\operatorname{tr}_{V_{1}} \cdot \overline{\operatorname{tr}_{V_{2}}} \cdot \operatorname{tr}_{V_{3}}$ whenever $\operatorname{tr}_{V_{1}}, \cdots, \operatorname{tr}_{V_{3}}$ are in $T_{0}\left(G_{\ell}\right)$.

Corollary 3.1.5. If $A$ is a QCG algebra then $A$ is a group algebra $\Leftrightarrow$ there exists a nonzero multiplicative linear functional on $A$ and every trace function $\operatorname{tr}_{V_{1}} \cdot \overline{\operatorname{tr}_{V_{2}}} \cdot \operatorname{tr}_{V_{3}}$ is a sum, with positive integer coefficients, of the primitive trace functions $\operatorname{tr}_{\left(G_{\ell}|E\rangle\right.}$ where $E$ are minimal left ideals in $A$.

Proof of 3.1.3. First we develop some general facts, valid for all QCG algebras. If $\lambda$ is a multiplicative linear functional on $A$ we must have $\|\lambda\|=1$. Let $N_{0}$ be the closed linear span of all minimal 
two-sided ideals in $A$ not contained in $\operatorname{Ker} \lambda$; then $\operatorname{dim} N_{0}=1$ and $\left(V^{0}, E_{0}\right)=\left(G_{\ell} \mid N_{0}\right)$ is irreducible. Now $\lambda$ has a unique extension to a multiplicative functional $\lambda$ on $A_{\zeta}^{m}$, in the sense that $\langle a, \lambda\rangle=\left\langle L_{a}, \lambda\right\rangle$ for $a \in A$ (see A.3.2), and this functional completely describes the action of the representation $\left(V^{0}, E_{0}\right)$ on $G_{\ell}$ since we can easily verify that $V_{g}^{0}(x)=\langle g, \lambda\rangle \cdot x$ for $x \in E_{0}=N_{0}$. If $(V, E) \in R\left(G_{\ell}\right)$ is equivalent to $\left(V^{0}, E^{0}\right)$ under an isomorphism $S: E_{0} \rightarrow E$, then $V^{0}{ }_{g}=S^{-1} \circ V_{g} \circ S$, which means $V_{g}^{0}(x)=\langle g, \lambda\rangle \cdot x=S^{-1}\left(V_{g}(S x)\right)$, so that $V_{g}^{0}(y)=\langle g, \lambda\rangle \cdot y$ for all $y \in E$. Notice in particular that any representation $(V, E) \sim$ $\left(V^{0}, E_{0}\right)$ acts on the subgroup $\Gamma_{\ell}=\left\{g \in G_{\ell}:\langle g, \lambda\rangle=1\right\}$ in $G_{\ell}$ as the one dimensional identity representation; this will be important later on.

If $\Gamma_{\ell}=\left\{g \in G_{\ell}:\langle g, \lambda\rangle=1\right\}$ then $\Gamma_{\ell}$ is a (so) closed normal subgroup in $G_{\ell}$ and we have an internal direct product factorization $G_{\ell}=S I \times \Gamma_{\ell}$, where $S I=\{\alpha I:|\alpha|=1\}$ and $I$ is the identity map on $A$ (see A.3.3 for detail). The behavior of $(V, E) \in R_{0}\left(G_{\ell}\right)$ when restricted to $\Gamma_{\ell}$ is of particular interest in proving 3.1.3. Denote the restriction of $(V, E)$ to $\Gamma_{\ell}$ as $\rho(V, E)=(\rho V, E)$. It is easy to see that the restriction map $\rho$ carries $R\left(G_{\ell}\right)$ into $R\left(\Gamma_{\ell}\right)$, preserving equivalences and the operations $\oplus, \otimes$, and *. Define $R_{0}\left(\Gamma_{\ell}\right)$ to be all representations in $R\left(\Gamma_{\ell}\right)$ equivalent to direct sums of representations $\left(\Gamma_{\ell} \mid E\right)$, where $E$ is a finite dimensional left ideal in $A$. Since a subspace of $A$ is $G_{\ell}$ invariant $\Leftrightarrow$ it is $\Gamma_{\ell}$ invariant, the proofs of 3.1.1 and 3.1.2 apply verbatim if we substitute $\Gamma_{\ell}$ for $G_{\ell}$ there.

LEMMA 3.1.6. On the set $R_{1}\left(G_{\ell}\right)=\left\{(V, E) \in R\left(G_{\ell}\right): V_{(\alpha g)}=\alpha V_{g}\right.$ for $\left.|\alpha|=1, g \in G_{\ell}\right\}$ the map $\rho$ is $1: 1$ and carries $R_{1}\left(G_{\ell}\right)$ onto $R\left(\Gamma_{\ell}\right)$, and the inverse $\left(\rho \mid R_{1}\left(G_{\ell}\right)\right)^{-1}$ preserves equivalences. Furthermore, $R_{0}\left(G_{\ell}\right) \subset R_{1}\left(G_{\ell}\right)$, and $R_{0}\left(\Gamma_{\ell}\right)$ has the properties

(1) Representation $(V, E) \in R\left(\Gamma_{\ell}\right)$ is in $R_{0}\left(\Gamma_{\ell}\right) \Leftrightarrow(V, E)=$ $\rho(W, F)$ for some $(W, F) \in R_{0}\left(G_{\ell}\right)$; hence $\rho\left(R_{0}\left(G_{\ell}\right)\right)=R_{0}\left(\Gamma_{\ell}\right)$.

(2) $R_{0}\left(\Gamma_{\ell}\right)$ contains all representations equivalent to the $n$ dimensional identity representation, for $n=1,2, \cdots$.

Proof. If $(V, E) \in R\left(\Gamma_{\ell}\right)$, define $\left(V^{\prime}, E\right) \in R\left(G_{\ell}\right)$ such that $V_{(\alpha \gamma)}^{\prime}=$ $\alpha V_{\gamma}$ for $|\alpha|=1$ and $\gamma \in \Gamma_{\ell}$; then if $g=\alpha \gamma \in G_{\ell}$ and $|\beta|=1$ we have $V_{(\beta g)}^{\prime}=V_{(\alpha \beta \gamma)}^{\prime}=\alpha \beta V_{\gamma}=\beta V_{g}^{\prime}$, so $\left(V^{\prime}, E\right) \in R_{1}\left(G_{\ell}\right)$, and clearly $\rho\left(V^{\prime}, E\right)=(V, E)$. The map $\rho$ is $1: 1$ because if $(\rho V)_{\gamma}=(\rho W)_{\gamma}$ for $\gamma \in \Gamma_{\ell}$ and $V, W \in R_{1}\left(G_{\ell}\right)$, then $V_{(\alpha \gamma)}=\alpha(\rho V)_{\gamma}=\alpha(\rho W)_{\gamma}=W_{(\alpha \gamma)}$ so that $V=W$. If $(V, E)$ and $(W, F)$ are equivalent in $R\left(\Gamma_{\ell}\right)$ under an isomorphism $S: E \rightarrow F$, their inverse images in $R_{1}\left(G_{\ell}\right)$ under $\rho$ are just $\left(V^{\prime}, E\right)$ and $\left(W^{\prime}, F\right)$ as above. Then we have $\left(V^{\prime}, E\right) \sim\left(W^{\prime}, F\right)$ since $V^{\prime}{ }_{(\alpha \gamma)}=\alpha V_{\gamma}=\alpha\left(S^{-1} \circ W_{\gamma} \circ S\right)=S^{-1} \circ \alpha W_{\gamma} \circ S=S^{-1} \circ W^{\prime}{ }_{(\alpha \gamma)} \circ S$.

Consider $(V, E)=\left(G_{\ell} \mid E\right) \in R_{0}\left(G_{\ell}\right)$ for some left ideal $E$ in $A$; 
clearly $V_{(\alpha g)}(x)=\alpha g(x)=\alpha V_{g}(x)$ for $g \in G_{\ell}$ and $|\alpha|=1$, so that $(V, E) \in R_{1}\left(G_{\ell}\right)$. It is easy to see that the same is true for all representations equivalent to direct sums of these, so $R_{0}\left(G_{\ell}\right) \subset R_{1}\left(G_{\ell}\right)$. Since $\rho\left(G_{\ell} \mid E\right)=\left(\Gamma_{\ell} \mid E\right)$ for finite dimensional left ideals in $A$ it is now obvious that $\rho\left(R_{0}\left(G_{\ell}\right)\right) \subset R_{0}\left(\Gamma_{\ell}\right)$. If $(V, E)$ is irreducible in $R_{0}\left(\Gamma_{\ell}\right)$ then $(V, E) \sim(W, F)=\left(\Gamma_{\ell} \mid F\right)$ for some finite dimensional minimal left ideal $F \subset A$, in view of (modified) 3.1.1, and $(W, F)=\rho\left(G_{\ell} \mid F\right) \in \rho\left(R_{0}\left(G_{\ell}\right)\right)$. But $\left(\rho \mid R_{1}\left(G_{\ell}\right)\right)^{-1}$ preserves equivalences, so $\rho\left(R_{0}\left(G_{\ell}\right)\right)$ is an equivalence closed subset of $R\left(\Gamma_{\ell}\right)$, and $(V, E) \in \rho\left(R_{0}\left(G_{\ell}\right)\right)$. Any $(V, E) \in R_{0}\left(\Gamma_{\ell}\right)$ is equivalent to a sum of irreducible representations in $R_{0}\left(\Gamma_{\ell}\right)$, as shown in (modified) 3.1.1, so it is a trivial matter to verify that $R_{0}\left(\Gamma_{\ell}\right) \subset \rho\left(R_{0}\left(G_{\ell}\right)\right)$; hence $R_{0}\left(\Gamma_{\ell}\right)=\rho\left(R_{0}\left(G_{\ell}\right)\right)$. Finally, if $\left(U, N_{0}\right)=$ $\left(\Gamma_{\ell} \mid N_{0}\right)$ ( $N_{0}$ was defined earlier) then $U_{\gamma}(x)=x$ for all $x \in N_{0}$, so $U$ is the one dimensional identity representation of $\Gamma_{\ell}$ and, clearly, $\left(U, N_{0}\right) \in R_{0}\left(\Gamma_{\ell}\right)$. This proves $(2)$.

LEMmA 3.1.7. If $R_{0}\left(G_{\ell}\right)$ is closed under the formation of $V_{1} \otimes\left(V_{2}\right)^{*} \otimes V_{3}$ for $V_{1}, \cdots, V_{3}$ in $R_{0}\left(G_{\ell}\right)$, then $R_{0}\left(\Gamma_{\ell}\right)$ is closed under the operations $\oplus, \otimes$, and $*$.

Proof. Closure under $\oplus$ is part of the definition of $R_{0}\left(\Gamma_{\ell}\right)$. Let $\left(U, N_{0}\right)=\left(\Gamma_{\ell} \mid N_{0}\right)$, the one dimensional identity representation, let $(V, E) \in R_{0}\left(\Gamma_{\ell}\right)$, and consider the inverse images in $R_{0}\left(G_{\ell}\right)$ under $\rho$ : $\left(U^{\prime}, N_{0}\right)$ and $\left(V^{\prime}, E\right)$. Then we have $W=U^{\prime} \otimes\left(V^{\prime}\right)^{*} \otimes U^{\prime} \in R_{0}\left(G_{\ell}\right)$, which $\Rightarrow \rho(W)=\left(\rho U^{\prime}\right) \otimes\left(\rho V^{\prime}\right)^{*} \otimes\left(\rho U^{\prime}\right)=U \otimes V^{*} \otimes U \in R_{0}\left(\Gamma_{\ell}\right) ;$ by looking at trace functions it is clear that $\rho W \sim V^{*}$, since $\operatorname{tr}_{\left(U, N_{0}\right)} \equiv 1$, so that $\left(V^{*}, E^{*}\right) \in R_{0}\left(\Gamma_{\ell}\right)$. Similarly if $(S, F),(T, E) \in R_{0}\left(\Gamma_{\ell}\right)$, with inverse images $\left(S^{\prime}, F\right),\left(T^{\prime}, E\right) \in R_{0}\left(G_{\ell}\right)$, under $\rho$, we have

$$
W=S^{\prime} \otimes\left(U^{\prime}\right)^{*} \otimes T^{\prime} \in R_{0}\left(G_{\ell}\right),
$$

which $\Rightarrow \rho(W)=S \otimes U^{*} \otimes T \sim S \otimes T$ is in $R_{0}\left(\Gamma_{\ell}\right)$, so $S \otimes T \in R_{0}\left(\Gamma_{\ell}\right)$.

Now we resume the proof of 3.1.3, beginning with $(\leftrightharpoons)$, by showing that $R_{0}\left(\Gamma_{\ell}\right)=R\left(\Gamma_{\ell}\right)$, and that this implies triviality of $N=\operatorname{Ker} T$ in the representation scheme for $A_{\ell}^{m}$ devised in 2.1.8 (recall $T: M\left(\Gamma_{\ell}\right) \rightarrow$ $A_{\ell}^{m}$ was defined so $\left.T \mu(a)=\int_{\Gamma \ell} \gamma(a) d \mu(\gamma)\right)$. Since $A \sim \varphi\left(L^{1}\left(\Gamma_{\ell}\right)\right)$ in the quotient algebra $M\left(\Gamma_{\ell}\right) / N$, it will follow that $A \sim L^{1}\left(\Gamma_{\ell}\right)$, as required. A representation function on a compact group $H$ is any function of the form $f_{\left(V, x, x^{*}\right)}(h)=\left\langle V_{h}(x), x^{*}\right\rangle$ where $x \in E, x^{*} \in E^{*}$, and $(V, E) \in R(H)$; we let $\mathscr{R}(H)$ indicate all representation functions and on $\Gamma_{\ell}$ we define $\mathscr{R}_{0}\left(\Gamma_{\ell}\right)=\left\{f_{\left(\nabla, x, x^{*}\right)}:(V, E) \in R_{0}\left(\Gamma_{\ell}\right), x \in E, x^{*} \in E^{*}\right\}$ within $\mathscr{R}\left(\Gamma_{\ell}\right)$. If we can prove that $\mathscr{R}_{0}\left(\Gamma_{\ell}\right)$ is a sup norm dense subalgebra of $C\left(\Gamma_{\ell}\right)$, 
it is not hard to show $R_{0}\left(\Gamma_{\ell}\right)=R\left(\Gamma_{\ell}\right)$ by using well-known orthogonality relations between functions in $\mathscr{R}\left(\Gamma_{\ell}\right)$. In fact if $R\left(\Gamma_{\ell}\right) \neq R_{0}\left(\Gamma_{\ell}\right)$ there is a nonzero irreducible $(V, E) \in R\left(\Gamma_{\ell}\right)$ which is not equivalent to any irreducible representation in $R_{0}\left(\Gamma_{\ell}\right)$ (see 3.1.1), and so if $f$ is a representation function arising from $(V, E)$, we get $\int_{\Gamma \ell} f(h) g(h) d h=0$ for all $g \in \mathscr{R}_{0}\left(\Gamma_{\ell}\right)$, which contradicts density of $R_{0}\left(\Gamma_{l}\right)$.

Lemma 3.1.8. $\mathscr{R}_{0}\left(\Gamma_{\ell}\right)$ is a sup norm dense subalgebra of $C\left(\Gamma_{\ell}\right)$.

Proof. By Stone-Weierstrass, we only have to show that $\mathscr{R}_{0}\left(\Gamma_{\ell}\right)$ separates points and is a conjugate closed subalgebra which contains all constant functions. But if $\Sigma \subset R(H)$ for a compact group $H$, then $\mathscr{R}_{\Sigma}(H)=\left\{f_{\left(V, x, x^{*}\right)}:(V, E) \in \Sigma\right\}$ is a conjugate closed subalgebra of $C(H)$ if $\Sigma$ is closed under equivalences and the operations $\oplus, \otimes$, and $*$ (see Chevalley [6], Ch. 6.7 and 6.8). The constant functions are the representation functions arising from the one dimensional identity representation in $R_{0}\left(\Gamma_{\ell}\right)$ so we only have to show $\mathscr{R}_{0}\left(\Gamma_{\ell}\right)$ separates points. For this it is clearly sufficient to show that $\Delta=\left\{\gamma \in \Gamma_{\ell}: V_{\gamma}=\right.$ $I_{E}$ for all $\left.(V, E) \in R_{0}\left(\Gamma_{\ell}\right)\right\}$ consists of just the unit $I \in \Gamma_{\ell}$. Suppose $\gamma \in \Delta$ then for every finite dimensional left ideal $E$ in $A$ we have $V_{\gamma}=I_{E}$ for $(V, E)=\left(\Gamma_{\ell} \mid E\right)$, which means $\gamma(x)=x$ for all $x \in E$, and hence for all $x \in \mathscr{A}(A)$. Since $\mathscr{A}(A)$ is norm dense we get $\gamma=I$, as required.

We show that $N=(0)$ if $R_{0}\left(\Gamma_{\ell}\right)=R\left(\Gamma_{\ell}\right)$ to complete the $(\Longleftrightarrow)$ part of 3.1.3. If $H$ is a compact group and $(V, E) \in R(H)$, we can induce a representation $(\boldsymbol{V}, E)$ on $M(H)$ with the property $\boldsymbol{V}_{\left(\delta_{h}\right)}=V_{h}$ by taking $\left\langle V_{\mu}(x V), x^{*}\right\rangle=\int_{H}\left\langle V_{h}(x), x^{*}\right\rangle d \mu(h)$ for $x \in E, x^{*} \in E^{*}$. The induced representation is bounded, has dense range, and is $(\sigma)$ continuous on norm bounded sets in $M(H)$ in the sense that

$$
\left\langle\boldsymbol{V}_{\mu_{j}}(x), x^{*}\right\rangle \longrightarrow\left\langle\boldsymbol{V}_{\mu}(x) x^{*}\right\rangle \text { if } \mu_{j} \stackrel{(\sigma)}{\longrightarrow} \mu
$$

and if $\left\|\mu_{j}\right\| \leqq M<\infty$. (See Loomis [11], Section 32). Now if $N \neq$ (0) it must contain a nonzero minimal left ideal $E_{0}$ in $L^{1}\left(\Gamma_{\ell}\right)$ because it is $(\sigma)$ closed. Consider $\left(V, E_{0}\right)=\left(\Gamma / \mid E_{0}\right)$; this representation is irreducible, and is known to be faithful on $E_{0}$, when extended to $M\left(\Gamma_{\ell}\right)$ (for this standard result see [11], $40 \mathrm{~B}$ and $40 \mathrm{C}$ ). Obviously $\left(V, E_{0}\right) \in R\left(\Gamma_{\ell}\right)$; however, if $\left(V, E_{0}\right) \in R_{0}\left(\Gamma_{\ell}\right)$ it is equivalent to $(W, J)=$ $(\Gamma \ell \mid J)$ for some finite dimensional minimal left ideal $J$ in $A$. Thus there is a linear isomorphism $S: E_{0} \rightarrow J$ such that $\gamma(\alpha)=W_{\gamma}(a)=$ $S \circ V_{\gamma} \circ S^{-1}(a)$ for $a \in J$. If $\mu \in N, a \in J$, and $x=S^{-1}(a)$ then 


$$
\begin{aligned}
0=T \mu(a) & =\int_{\Gamma \ell} \gamma(a) d \mu(\gamma) \\
& =\int_{\Gamma \ell} S\left(V_{\gamma}(x)\right) d \mu(\gamma) \\
& =S\left(V_{\mu}(x)\right)
\end{aligned}
$$

so that $V$ annihilates $N$ when extended to $M\left(\Gamma_{\ell}\right)$, a contradiction. Thus $N=(0)$ and $(\Longleftrightarrow)$ is proved.

For the converse proof we must show $V_{1} \otimes\left(V_{2}\right)^{*} \otimes V_{3} \in R_{0}\left(G_{\ell}\right)$ whenever $V_{1}, \cdots, V_{3} \in R_{0}\left(G_{\ell}\right)$, if $A=L^{1}(H)$ for some compact group $H$. Let us identify $A_{\ell}^{m} \sim M(H)$ and define the nonzero multiplicative functional $\lambda$ on $A$ (and $A_{\ell}^{m}$ ) by taking $\left\langle L_{\mu}, \lambda\right\rangle=\int_{H} d \mu$. Clearly $G_{\ell}=$ $\left\{\alpha L\left(\delta_{h}\right):|\alpha|=1, h \in H\right\}$ and $\Gamma_{\ell}=\left\{g \in G_{\ell}:\langle g, \lambda\rangle=1\right\}$ is just $\left\{L\left(\delta_{h}\right): h \in H\right\}$; thus $G_{\ell}=S I \times \Gamma /$ and the discussion which preceeds the $\Longleftrightarrow$ proof applies to $R_{0}\left(\Gamma_{\ell}\right)$ and $R_{0}\left(G_{\ell}\right)$. We assert that $R_{0}\left(\Gamma_{\ell}\right)=R\left(\Gamma_{\ell}\right)$; the reader can easily verify this by using the well known fact that any continuous irreducible representation of $H$ is equivalent to the action of $H$ by left translation on some minimal left ideal in $A=L^{1}(H)$. Notice that $\tau: h \rightarrow L\left(\delta_{h}\right)$ is a topological isomorphism between $H$ and $(\Gamma \ell,(s o))$.

Applying 3.1.6 we see that $R\left(\Gamma_{\ell}\right)=R_{0}\left(\Gamma_{\ell}\right)=\rho\left(R_{0}\left(G_{\ell}\right)\right)$ where $\rho$ is the map restricting representations of $G_{\ell}$ to $\Gamma_{\%}$ In 3.1 .6 we showed $R_{1}\left(G_{\ell}\right) \supset R_{0}\left(G_{\ell}\right)$ and that $\rho$ carries $R_{1}\left(G_{\ell}\right)$ and $R_{0}\left(G_{\ell}\right) 1: 1$ onto $R\left(\Gamma_{\ell}\right)$ and $R_{0}\left(\Gamma_{\ell}\right)$ respectively; since $R_{0}\left(\Gamma_{\ell}\right)=R\left(\Gamma_{\ell}\right)$ we see that $R_{0}\left(G_{\ell}\right)=$ $R_{1}\left(G_{\ell}\right)$. Thus $(V, E) \in R_{0}\left(G_{\ell}\right) \Leftrightarrow V_{(\alpha I)}=\alpha V_{I}=\alpha I_{E}$, all $|\alpha|=1$.

It is easy to see that $V_{(\alpha I)}=\alpha V_{I}$ for $|\alpha|=1 \Leftrightarrow \operatorname{tr}_{V}(\alpha I)=\alpha \cdot \operatorname{dim}[E]$ for all $|\alpha|=1$; hence this is necessary and sufficient condition for $(V, E) \in R_{0}\left(G_{\ell}\right)$. If $(W, E) \in R_{0}\left(G_{\ell}\right)$ then we have $\left(W^{*}\right)_{(\alpha \gamma)}=\bar{\alpha}\left(W^{*}\right)_{\gamma}$ since

$$
\begin{aligned}
\left\langle x,\left(W^{*}\right)_{a \gamma} x^{*}\right\rangle & =\left\langle x,\left(W_{(\alpha \gamma)-1}\right)^{*} x^{*}\right\rangle=\left\langle\bar{\alpha} W_{\gamma-1}(x), x^{*}\right\rangle \\
& =\left\langle\bar{\alpha} x,\left(W_{\gamma-1}\right)^{*} x^{*}\right\rangle=\left\langle x, \bar{\alpha}\left(W_{\gamma-1}\right)^{*} x^{*}\right\rangle=\left\langle x, \bar{\alpha}\left(W^{*}\right)_{\gamma}(x)\right\rangle
\end{aligned}
$$

for all $x \in E, x^{*} \in E^{*}$. This implies that, in particular, $\left(W^{*}\right)_{\alpha I}=$ $\bar{\alpha}\left(W^{*}\right)_{I}=\bar{\alpha} I_{E}$ whenever $|\alpha|=1$, so that $\operatorname{tr}_{\left(W^{*}\right)}(\alpha I)=\bar{\alpha} \operatorname{dim}[E]$. Now if $\left(V^{i}, E_{i}\right)$ are in $R_{0}\left(G_{\ell}\right)$ for $i=1,2,3$ and if $|\alpha|=1$ we have

$$
\begin{aligned}
\operatorname{tr}_{V^{1} \otimes\left(V^{2}\right) * \otimes V^{3}}(\alpha I) & =\operatorname{tr}_{V^{1}}(\alpha I) \cdot \operatorname{tr}_{\left(V^{2}\right) *}(\alpha I) \cdot \operatorname{tr}_{V^{3}}(\alpha I) \\
& =\alpha \cdot \operatorname{dim}\left[E_{1}\right] \cdot \bar{\alpha} \operatorname{dim}\left[E_{2}\right] \cdot \alpha \operatorname{dim}\left[E_{3}\right] \\
& =\alpha \operatorname{dim}\left[E_{1}\right] \operatorname{dim}\left[E_{2}\right] \operatorname{dim}\left[E_{3}\right]
\end{aligned}
$$

and $\operatorname{dim}\left[E_{1}\right] \cdot \operatorname{dim}\left[E_{2}\right] \cdot \operatorname{dim}\left[E_{3}\right]$ is just the dimension of the representation space of $V^{1} \otimes\left(V^{2}\right)^{*} \otimes V^{3}$. Thus $V^{1} \otimes\left(V^{2}\right)^{*} \otimes V^{3} \in R_{0}\left(G_{\ell}\right)$, as required to prove $(\Longrightarrow)$ in 3.1 .3 . 
If $A$ is a commutative QCG algebra it has many nonzero multiplicative functionals ( $A$ is semi-simple) and all irreducible representations of $G(A)$ are one dimensional. It is easy to identify the traces of these representations in $R_{0}(G)$ with the set of (so) continuous multiplicative characters $\alpha_{M}(g)=\langle g, M\rangle$ obtained when we extend the nonzero multiplicative functionals on $A$, corresponding to $M \in \mathscr{C}_{A}$, to $A^{m}$. This proves:

Corollary 3.1.9. $A$ is a commutative QCG algebra then $A$ is a group algebra $\Leftrightarrow$ the set $R=\left\{\alpha_{M}: M \in \mathscr{l}_{A}\right\} \subset G^{\wedge}$ contains all functions $\lambda(g)=\alpha_{M_{1}}(g) \overline{\alpha_{M_{2}}(g)} \alpha_{M_{3}}(g)$ whenever $\alpha_{M_{1}}, \cdots, \alpha_{M_{3}} \in R$.

Evidently $R$ has this property $\Leftrightarrow$ it is a coset of some subgroup in $G^{\wedge}$. This should be compared with the results of Section 2.3.

3.2. Involutions in QCG algebras. Since group algebras are endowed with natural involutions, one might hope to reduce the complexity of the preceding identification by trying instead to identify group algebras as QCG algebras which have a "nice" involution. This hope is unfounded: although the notion of involution never appears in the definition and characterization of QCG algebras, every QCG algebra $A$ has a natural symmetric involution $\left(-a^{*} a\right.$ quasi-regular in $A$ for every $a \in A$; see [14], 4.1 and 4.7). This involution has an abstract characterization. We will only state these results and indicate the general line of reasoning, since they are of secondary interest.

If $A$ is QCG we have a concrete realization $A \sim \varphi\left(L^{1}(H)\right)$ in $M(H) / N$ (and $\left.A_{\zeta}^{m} \sim M(H) / N\right)$, where $H$ is a compact group and $N$ a $(\sigma)$-closed two-sided ideal in $M(H)$. Then the natural involution $(*)$ on $M(H)$ gives an involution on $A$ (and on $A_{\ell}^{m}$ ) because, as is easily verified, $N$ must be $*$ self adjoint. Let us define the "natural involution" (*) on $A$ and $A_{\ell}^{m}$ to be the involution obtained by considering $H=\left(G_{\ell},(s o)\right)$ and the representation $T: M\left(G_{\ell}\right) \rightarrow A_{\ell}^{m}$ defined in 2.1.4; thus $(T \mu)^{*}=T\left(\mu^{*}\right)$ for $\mu \in M\left(G_{\ell}\right)$.

It is helpful to look into the relationship between involutions on $A$ and on $A_{\zeta}^{m}$. Let us say that $(\#)$ on $A_{\zeta}^{m}$ is an extension of $\left(\xi^{2}\right)$ on $A$ if $\left(L_{a}\right)^{\sharp}=L_{\left(a^{\sharp}\right)}$ for $a \in A$. If $A$ is commutative we get at least one extension by taking $F^{\sharp}(a)=\left(F\left(a^{\vec{r}}\right)\right)^{\text {) }}$ for $F \in A_{\ell}^{m}, a \in A$. Such extensions are not easily constructed if $A$ is noncommutative; they do exist for QCG algebras because QCG algebras have the special property that $A_{\ell} \subset A_{\ell}^{m}$ is a two-sided ideal (rather than just a left ideal). This follows since $L^{1}\left(G_{\ell}\right)$ is two-sided in $M\left(G_{\ell}\right)$.

THEOREM 3.2.1. If (污) is an isometric involution on QCG algebra $A$, it has a unique isometric extension ( $\left.\xi^{3}\right)$ to $A_{\zeta}^{m}$. 
The proof uses the concrete representation theory. Evidently the natural involutions (*) on $A, A_{\ell}^{m}$ are isometric and $(*)$ on $A_{\ell}^{m}$ is the unique extension of $(*)$ on $A$.

THEOREM 3.2.2. An isometric involution ( 2 ) on QCG algebra $A$ is the natural involution on $A \Leftrightarrow$ its extension ( $\xi^{3}$ to $A_{l}^{m}$ is such that $g^{\text {茨 }}=g^{-1}$ for all $g \in G_{\ell}$.

Obviously the natural involution $(*)$ on $A_{\ell}^{m}$ has this property. For $(\Longleftrightarrow)$ one first proves that any isometric involution $(\xi)$ such that $g^{\text {席 }}=g^{-1}$ must map $A_{\ell}$ onto $A_{\ell}$ (hence it is associated with an involution on $\left.A \sim A_{\ell}\right)$. This fact can be used to show that any such involution on $A_{\ell}^{m}$ is (so) bicontinuous; since $\left(\Sigma^{2}\right) \equiv(*)$ on $G_{\ell}$ the same is true for co $\left[G_{\ell}\right]$, and $($ so $)$ density gives $(\hat{\xi})=(*)$ on $\Sigma_{A_{\ell}^{m}}$ as required.

Now it is a fairly simple matter to prove:

THEOREM 3.2.3. The natural involution in any QCG algebra is symmetric.

It suffices to show $a a^{*}=0 \Rightarrow a=0$ (see [14], 4.10.11).

REMARK. If we realize $A \sim \varphi\left(L^{1}(H)\right), A_{\ell}^{m} \sim M(H) / N$ where $H$ is any compact group, $N \subset M(H)$ a $(\sigma)$-closed two-sided ideal, and $\varphi: M(H) \rightarrow M(H) / N$ the quotient map, then $N$ is $(\xi)$ self-adjoint with respect to the involution ( $s)$ in $M(H)$. It is a fairly straightforward matter to prove that the involution $(\Sigma)$ induced on $A_{\ell}^{m}$ always has the property $g^{\text {㞤 }}=g^{-1}$; hence it coincides with the natural involution (*) (which was defined in terms of a specific realization of $A_{\ell}^{m} ; A$ ). Thus the natural involution is canonical in that it does not depend on which concrete representation we look at.

\section{Appendix}

A.1. General facts about group and measure algebra. Let $H$ be a compact group and define the operator topology $(s o)_{\zeta}$ in $M(H)$ as indicated in 1.2. The following results are equally valid for the right-handed operator topology $(s o)_{r}$ after a few simple alterations in the proofs.

THEOREM A.1.1. Let $(\sigma)$ be the weak * topology on $M(H)$, then the topologies $(s o)_{\ell}$ and $(\sigma)$ coincide on norm bounded sets in $M(H)$.

Proof. Let $\mathscr{H}=\mathscr{E}_{M(H)}$ and $\mathscr{H}_{0}=\left\{\delta_{h}: h \in H\right\}$, then it is easy to see 
that these topologies coincide on $\mathscr{H}$ and $\mathscr{H}{ }_{0}$, making them topologically isomorphic to $S \times H$ and $H$ respectively; in particular these sets are compact. From [7], p. 511 we see that co[ $\left.\mathscr{H C}:(s o)_{\ell}\right]$ must be $\left(\right.$ so $_{\ell}$ compact, as is $\operatorname{co}[\mathscr{H}:(\sigma)]=\Sigma_{M(H)}$ in the $(\sigma)$ topology. The identity map $\left.j:(M(H)),(s o)_{\ell}\right) \rightarrow(M(H),(\sigma))$ is continuous on norm bounded sets; in fact, if $\Psi \in C(H)$ then there exists $f \in L^{1}(H)$ corresponding to $\varepsilon>0$ such that $\|f\|=1$ and $\left|\int_{H} \Psi(s t) f(t) d t-\Psi(s)\right|<\varepsilon / 3$ for all $s \in H$. If $\left\{\mu_{j}: j \in J\right\}$ is a net in $\Sigma_{M(H)}$ with $\mu_{j} \stackrel{(s o)}{\longrightarrow} \mu$, then $\left|\left\langle\mu_{j} * f, \Psi\right\rangle-\left\langle\mu_{j}, \Psi\right\rangle\right|<\varepsilon / 3$ for all $j \in J$, and likewise for $\mu$, so that (for large $j$ ) $\left|\left\langle\mu_{j}, \Psi\right\rangle-\langle\mu, \Psi\rangle\right|\langle\varepsilon$, as required.

Now we see that $\operatorname{co}\left[\mathscr{H}:(s o)_{\ell}\right]$ is $(\sigma)$ compact, and it obviously contains $\operatorname{co}[\mathscr{C}:(\sigma)]=\Sigma_{M(H)}$; but clearly $\mu \in \operatorname{co}\left[\mathscr{C}:(s o)_{\ell}\right] \Rightarrow\|\mu\| \leqq 1$, which gives reverse containment. It is obvious that the topologies coincide on $\Sigma_{M(H)}$ since they are comparable and since $\Sigma_{M(H)}$ is compact in each topology.

THEOREM A.1.2. Let $H$ be a locally compact group and let $N \subset M(H)$ be a $(\sigma)$ closed subspace. If $\varphi: M(H) \rightarrow M(H) / N$ is the canonical linear map, and $M(H) / N$ is given the quotient norm, then $\varphi\left(\Sigma_{M(H)}\right)=\Sigma_{M(H) / N}$.

Proof. Since $\alpha=\|\phi \mu\|=\inf \{\|\mu+n\|: n \in N\}$ it is clear that, for $n=1,2 \cdots$ there exist $\mu_{n} \in \mu+N$ such that $\left\|\mu_{n}\right\| \leqq \alpha+1 / n$. Now $\left\{\left\|\mu_{n}\right\|: n=1,2 \cdots\right\}$ is bounded, so there is a $(\sigma)$ convergent subnet $\mu_{n(k)} \stackrel{(\sigma)}{\longrightarrow} \lambda \in M(H)$; clearly $\lambda \in \mu+N$, since $N$ is $(\sigma)$ closed (hence $\varphi \lambda=\varphi \mu)$, and $\|\lambda\| \leqq \limsup \left\{\left\|\mu_{n(k)}\right\|: k \in K\right\} \leqq \alpha$, which $\Longrightarrow\|\lambda\|=$ $\|\varphi \mu\|$ as required.

It is well known that every group algebra has a minimal twosided approximate identity. The following stronger result holds for compact groups.

THEOREM A.1.3. If $A$ is the group algebra of a compact group $H$, then $A$ has a minimal central approximate identity.

Proof. Since $H$ is compact there is a neighborhood basis $\left\{V_{j}: j \in J\right\}$ of the unit $e \in H$, such that $y^{-1} V_{j} y=V_{j}=V_{j}^{-1}$ for all $j \in J, y \in H$. If we take any basis $\left\{U_{j}: j \in J\right\}$ such that $U_{j}=U_{j}^{-1}$, we get the desired basis as $V_{j}=\bigcap\left\{y^{-1} U_{j} y: y \in H\right\}$ (see Montgomery-Zippin [12], Section 2.4). Take $e_{j}=\chi_{V_{j}} / m\left(V_{j}\right)$, where $\chi_{E}$ is the characteristic function of $E$; it is easy to check that $e_{j}$ is central in $L^{1}(H)$, so $\left\{e_{j}\right\}$ is the desired approximate identity. 
A.2. Dual algebras with approximate identities. As is well known, a semisimple dual Banach algebra with an identity is finite dimensional.

THEOREM A.2.1. If $A$ is a semi-simple dual Banach algebra with a central minimal approximate identity then the structure theory of Kaplansky [10] applies and all minimal closed two-sided ideals are finite dimensional.

Proof. If $M$ is a minimal closed two-sided ideal in $A$ and if $\left\{e_{\alpha}: \alpha \in Q\right\}$ is a maximal collection of minimal idempotents in $M$ which are pairwise orthogonal $\left(e_{\alpha} e_{\beta}=e_{\beta} e_{\alpha}=0\right.$ if $\left.\alpha \neq \beta\right)$, then $e_{\alpha} A e_{\beta}$ is always a one dimensional subspace of $A$ and we can find a collection of matrix units in $M,\left\{e_{\alpha \beta} \in e e_{\alpha} A \in_{\beta}: \alpha, \beta \in Q\right\}$ such that $e_{\alpha \alpha}=e_{\alpha}$ and $e_{\alpha \beta} e_{\mu \lambda}=\delta_{\beta \mu} e_{\alpha \lambda}$. The algebra $M_{0}=\left\{\Sigma \xi_{\alpha \beta} e_{\alpha \beta}\right.$ (finite sums) $: \xi_{\alpha \beta}$ complex $\}$ is a two-sided ideal of $A$, norm dense in $M$. If $x \in A$ is central then $x y=\lambda(x) y$ for all $y \in M$; in fact $x e_{\alpha \alpha}=e_{\alpha \alpha} x e_{\alpha \alpha} \in e_{\alpha} A e_{\alpha}$, which implies that $x e_{\alpha \alpha}=$ $\lambda_{\alpha}(x) e_{\alpha}$, since $e_{\alpha} A e_{\alpha}$ is one dimensional. But $x e_{\alpha \beta}=x e_{\alpha} \alpha_{\alpha \beta} e_{\beta}$ for some $a_{\alpha \beta} \in A$, so $x e_{\alpha \beta}=\lambda_{\alpha}(x) e_{\alpha} \cdot a_{\alpha \beta} \cdot e_{\beta}=\lambda_{\alpha}(x) e_{\alpha \beta}$ and also $x e_{\alpha \beta}=x e_{\alpha} a_{\alpha \beta} e_{\beta}=$ $e_{\alpha} a_{\alpha \beta} x e_{\beta}=\lambda_{\beta}(x) e_{\alpha \beta}$; hence $\lambda_{\alpha}(x)=\lambda_{\beta}(x)=\lambda(x)$ for all $\alpha, \beta \in Q$ and this implies that $x y=\lambda(x) y$ whenever $y \in M\left(M_{0}\right.$ is dense in $\left.M\right)$. Now there is at least one $x \in Z(A)$ (center of $A$ ) such that $x M \neq(0)$, since $Z(A)$ contains an approximate identity for $A$. By properly scaling $x \in Z(A)$ we can insure that $x y=y$ for all $y \in M$.

According to Kaplansky [10], Theorem 5, if $\left\{M_{\mu}: \mu \in J\right\}$ are the minimal closed two-sided ideals in $A$, we have $M_{\mu} M_{\lambda}=M_{\lambda} M_{\mu}=(0)$ if $M_{\mu} \neq M_{\lambda}$ and the algebraic (direct) sum $\sum \oplus\left\{M_{\mu}: \mu \in J\right\}$ is a norm dense two-sided ideal in $A$. With $x \in Z(A)$ chosen for (fixed) ideal $M$, as above, we can find elements $x_{n}=f_{n}+\sum\left\{\xi_{\alpha \beta}(n) e_{\alpha \beta}: \alpha, \beta \in Q\right\}$ such that $\left\|x-x_{n}\right\| \rightarrow 0$, where $f_{n} \in \sum \oplus\left\{M_{\mu}: \mu \in J ; M_{\mu} \neq M\right\}$ and $\sum \xi_{\alpha \beta}(n) e_{\alpha \beta}$ is a finite sum in $M_{0}$. Let $n$ be chosen such that $\left\|x-x_{n}\right\|<1 / 2$. If $\operatorname{dim}[M]=\infty$ them $\operatorname{dim}\left[M_{0}\right]=\infty$ and there exist indices $\left\{\alpha_{1}, \cdots, \alpha_{m}\right\}$ in $Q$ such that $\xi_{\alpha \beta}(n)=0$ for all $\beta \in Q$ if $\alpha \neq \alpha_{1}, \cdots, \alpha_{m}$. For any such index $\alpha \in Q$ we have

$$
\frac{1}{2}\left\|e_{\alpha \alpha}\right\| \geqq\left\|e_{\alpha \alpha}\left(x-x_{n}\right)\right\|=\left\|e_{\alpha \alpha}-e_{\alpha \alpha} f_{n}-\sum\left\{\xi_{\alpha \beta}(n) e_{\alpha \beta}: \beta \in Q\right\}\right\| \text {. }
$$

But clearly, $0=\sum\left\{\xi_{\alpha \beta}(n) e_{\alpha \beta}: \beta \in Q\right\}$ and $e_{\alpha \alpha} f_{n}=0$, so that $1 / 2\left\|e_{\alpha \alpha}\right\| \geqq$ $\left\|e_{\alpha \alpha}\right\| \geqq 1$, a contradiction. Thus $\operatorname{dim}[M]<\infty$ as required.

A.3. Elementary properties of multipliers and translations on Banach algebras. Consider an arbitrary Banach algebra $A$ with group of left translations $G_{\ell}=G_{\ell}(A)$, given the (so) topology. All of the 
following results have right-handed counterparts which we do not state explicitly.

THEOREM A.3.1. The translations $G_{\ell}$ form a Hausdorff topological group. The extreme points $\mathscr{E}_{\Delta_{\zeta}^{m}}$ contain $G_{\ell}$ and $\mathscr{E}_{\Delta_{\zeta}}=G_{\zeta}$ if these extreme points form a group.

REMARK. In [3] it is shown that, if a Banach algebra $B$ has an identity $I$ with $\|I\|=1$, then $I$ is an extreme point in $\Sigma_{B}$.

Proof. The Hausdorff property is obvious, and if $\left\{g_{j}: j \in J\right\}$ and $\left\{h_{k}: k \in K\right\}$ are nets in $G_{\ell}$, both (so) convergent to $I$, then

$$
\begin{aligned}
\left\|g_{j}\left(h_{k}(a)\right)-a\right\| & \leqq\left\|g_{j}\left(h_{k}(a)\right)-g_{j}(a)\right\|+\left\|g_{j}(a)-a\right\| \\
& =\left\|h_{k}(a)-a\right\|+\left\|g_{j}(a)-a\right\| \longrightarrow 0
\end{aligned}
$$

for $a \in A$, proving joint (so) continuity of multiplication. Inversion is also (so) continuous since

$$
\left\|g_{j}^{-1}(a)-a\right\|=\left\|g_{j}\left(g_{j}^{-1}(a)\right)-g_{j}(a)\right\|=\left\|a-g_{j}(a)\right\| \longrightarrow 0 .
$$

If $g \in G_{\ell}$ it has an inverse $g^{-1} \in G_{\ell}$, and if we can decompose $g=$ $\alpha F_{1}+(1-\alpha) F_{2}$, with $0<\alpha<1$ and $F_{1}, F_{2} \in \Sigma_{A^{m}}$, then $I=\alpha g^{-1} F_{1}+$ $(1-\alpha) g^{-1} F_{2}$; since $\left\|g^{-1} F_{i}\right\| \leqq 1$ we get $g^{-1} F_{1}=g^{-1} F_{2}$ and $F_{1}=F_{2}$, so $G_{\iota} \subset \mathscr{E}_{A_{\ell}^{m}}$. If $\mathscr{E}_{A^{m}}$ is a group (with unit $I$ ), then $T(A)=A$ if $T \in \mathscr{E}_{4}{ }_{\ell}^{m}$ and $\|T a\|=\|a\|$ on $A$; otherwise $\|T a\|<\|a\|$ for some $a \in A$ and $\|a\|=\left\|T^{-1}(T a)\right\| \leqq\left\|T^{-1}\right\| \cdot\|T a\|=\|T a\|<\|a\|$.

If $A$ is commutative and if $\psi$ is a multiplicative linear functional on $A$, then $\psi$ extends uniquely to $\Psi$ on $A^{m}$ in the sense that $\left\langle L_{a}, \Psi\right\rangle=$ $\langle a, \psi\rangle$ for all $a \in A$; in fact if $F \in A^{m}$ we take $\langle F, \Psi\rangle=\langle F(a), \psi\rangle\langle a, \psi\rangle$ for any $a \in A$ such that $\psi(a) \neq 0$ ( $\psi=0$ extends trivially). Things are not so easy if $A$ fails to be commutative.

THEOREM A.3.2. If $A$ has a two-sided approximate identity $\left\{e_{j}: j \in J\right\}$ then any multiplicative linear functional $\psi$ on $A$ extends uniquely to $A_{\zeta}^{m}$. The extension is given by the formula $\langle F, \Psi\rangle=$ $\langle F(a), \psi\rangle\langle\langle a, \psi\rangle$, where $a \in A$ is any element such that $\psi(a) \neq 0$.

Proof. Since

$$
\begin{aligned}
\psi(b) \psi(F a) & =\psi(F(a) \cdot b)=\psi(F(a b)) \longleftrightarrow \psi\left(F\left(e_{j} a b\right)\right)=\psi\left(\left(F e_{j}\right) a b\right) \\
& =\psi\left(\left(F e_{j}\right) b a\right)=\psi\left(F\left(e_{j} b a\right)\right) \longrightarrow \psi(F(b a))=\psi(F b) \psi(a)
\end{aligned}
$$

we see that $\Psi$ is well defined. Now let $F_{1}, F_{2} \in A_{\swarrow}^{m}$; if for some $a \in A$ 
we have $\langle a, \psi\rangle \neq 0$ and $\left\langle F_{2}(a), \psi\right\rangle \neq 0$, then $\left\langle F_{1} F_{2}, \Psi\right\rangle=\left[\left\langle F_{1} F_{2}(a), \psi\right\rangle\left\langle F_{2} a, \psi\right\rangle\right]\left[\left\langle F_{2} a, \psi\right\rangle\langle\langle a, \psi\rangle]=\left\langle F_{2}, \Psi\right\rangle \cdot\left\langle F_{1}, \Psi\right\rangle\right.$.

However, if $\left\langle F_{2} a, \psi\right\rangle=0$ whenever $\langle a, \psi\rangle \neq 0$ then $\left\langle F_{2}, \Psi\right\rangle=0$ and, for any such $a \in A$, we have

$$
\begin{aligned}
\left\langle F_{1} F_{2}(a), \psi\right\rangle \longleftarrow & \left\langle F_{1}\left(e_{j} F_{2}(a)\right), \psi\right\rangle=\left\langle\left(F_{1} e_{j}\right)\left(F_{2} a\right), \psi\right\rangle \\
& =\left\langle F_{1}\left(e_{j}\right), \psi\right\rangle \cdot\left\langle F_{2}(a), \psi\right\rangle=0 ;
\end{aligned}
$$

hence, $\left\langle F_{1} F_{2}, \Psi\right\rangle=0=\left\langle F_{1}, \Psi\right\rangle \cdot\left\langle F_{2}, \Psi\right\rangle$, and $\Psi$ is multiplicative, as well as linear. Clearly $\left\langle L_{a}, \Psi\right\rangle=\langle a, \psi\rangle$ if $a \in A$. Uniqueness follows since we must have

$$
\begin{aligned}
\langle F(a), \psi\rangle & =\left\langle L_{F^{(a)}}, \Psi\right\rangle=\left\langle F \circ L_{a}, \Psi\right\rangle \\
& =\langle F, \Psi\rangle \cdot\left\langle L_{a}, \Psi\right\rangle=\langle F, \Psi\rangle \cdot\langle a, \Psi\rangle
\end{aligned}
$$

for all $a \in A, f \in A_{\ell}^{m}$.

The usefulness of A.3.2 stems from the fact that existence of a multiplicative functional in $A_{\zeta}^{m}$ which does not vanish on $A_{\ell}$ implies the existence of an internal direct product factorization of $G_{\ell}=$ $S I \times \Gamma_{\ell}$, where $\Gamma_{\ell}$ is some (so) closed normal subgroup.

THEOREM A.3.3. Let $\psi$ be a multiplicative functional on $A_{\ell}^{m}$ which does not vanish on $A_{\ell}$, and define $\alpha_{\psi}(g)=\langle g, \psi\rangle$ on $G_{\ell}$. Then $\alpha_{\psi}$ is a (so) continuous multiplicative character on $G_{\ell}$, the subgroups $\Gamma_{\ell}=\left\{g \in G_{\ell}:\langle g, \psi\rangle=1\right\}$ and $S I=\{\alpha I:|\alpha|=1\}$ are (so) closed normal subgroups which generate $G_{\ell}$, and $S I \cap \Gamma_{\ell}=\{I\}$ so that $G_{\ell}=S I \times \Gamma_{\ell}$. If $G_{\ell}$ can be factored as an internal direct product $G_{\ell}=S I \times \Gamma$ for some (so) closed normal subgroup $\Gamma$, then the natural projections $\pi_{\Gamma}: g \rightarrow \gamma$ and $\pi_{S I}: g \rightarrow \alpha I$ (if $g=(\alpha I, \gamma)$ in the direct product) are (so) continuous homomorphisms, and the elements of $\Gamma$ are pairwise linearly independent in $A_{\zeta}^{m}$. Furthermore if $G_{\ell}=S I \times \Gamma_{i}(i=1,2)$ are two such factorizations then the map $g \rightarrow \overline{\pi_{(S)_{2}}(g) \cdot g}$ is a topological automorphism of $G_{\ell}$ which maps $\Gamma_{1}$ onto $\Gamma_{2}$; hence the decomposition $G_{\ell}=S I \times \Gamma$ is essentially unique.

Proof. The function $\alpha_{\psi}$ is clearly unimodular and multicative on $G_{\ell}$. If $a \in A$ with $\left\langle L_{a}, \psi\right\rangle \neq 0$, and if $g_{j} \stackrel{(s o)}{\longrightarrow} g$ in $G_{\ell}$, then

$$
\left\|g_{j} \circ L_{a}-g \circ L_{a}\right\|=\left\|g_{j}(a)-g(a)\right\| \longrightarrow 0,
$$

so that $\left\langle g_{j}, \psi\right\rangle \cdot\left\langle L_{a}, \psi\right\rangle=\left\langle g_{j} L_{a}, \psi\right\rangle \rightarrow\left\langle g L_{a}, \psi\right\rangle=\langle g, \psi\rangle \cdot\left\langle L_{a}, \psi\right\rangle$, and this in turn implies that $\left\langle g_{j}, \psi\right\rangle \rightarrow\langle g, \psi\rangle$. Thus it is clear that $S I$ 
and $\Gamma_{\ell}$ are (so) closed normal subgroups in $G_{\ell}$. Furthermore, $S I \cap \Gamma_{\ell}=$ $\{I\}$, since $\langle\alpha I, \psi\rangle=\alpha\langle I, \Psi\rangle=\alpha$, and it is clear that $\{S I\} \cdot \Gamma_{\ell}=G_{\ell}$.

If $G_{\ell}=S I \times \Gamma$, let $\left\{g_{j}=\alpha_{j} \gamma_{j}: j \in J\right\}$ be a net in $G_{\ell}$ such that $g_{j} \stackrel{(s o)}{\longrightarrow} g=\alpha \gamma$. If $\beta$ is any limit point of the net of scalars $\left\{\alpha_{j}\right\}$ in the circle, there is a subnet $\left\{\alpha_{j(k)}\right\}$ such that $\left|\alpha_{j(k)}-\beta\right| \rightarrow 0$ while $\alpha_{j(k)} \gamma_{j(k)} \stackrel{(s o)}{\longrightarrow} \alpha \gamma$; hence $\gamma_{j(k)} \stackrel{(s o)}{\longrightarrow}(\alpha / \beta) \gamma \in \Gamma$. But if $(\alpha / \beta) \gamma \in \Gamma$ then we must have $\alpha=\beta$, so $\alpha_{j} \rightarrow \alpha$ and $\gamma_{j} \stackrel{(s o)}{\longrightarrow} \gamma$, as required for continuity of the projection homomorphisms in $G_{\ell}=S I \times \Gamma$. If, in such a factorization, we should have $\gamma_{1}, \gamma_{2} \in \Gamma$ linearly dependent in $A_{\ell}^{m}$, then $\alpha \gamma_{1}=\gamma_{2}$ for (unimodular) complex scalar $\alpha$. Since $\gamma_{1}, \gamma_{2} \in \Gamma$ this means $\alpha=1$. The verifications in the last part of A.3.3 are straightforward.

A.4. Strong operator topologies. Let $A$ be a Banach space and (so) the strong operator topology in $B(A, A)$.

LEMMA A.4.1. With respect to the (so) topology, any closed bounded subset $E \subset B(A, A)$ is complete.

Proof. Set $E$ is bounded in the (so) topology $\Leftrightarrow\{T(x): T \in E\}$ is norm bounded in $A$ for each $x \in A$. If $\left\{T_{j}: j \in J\right\}$ is any (so)-Cauchy net in $E$ and $x \in A$, then $\left\{T_{j}(x): j \in J\right\}$ is a bounded Cauchy net in the Banach space $A$, so there exists $T(x) \in A$ such that $T_{j}(x) \stackrel{\text { norm }}{\longrightarrow} T(x)$. Obviously $T: A \rightarrow A$ is linear; since $\left\{T_{j}(x): j \in J\right\}$ is norm bounded for each $x \in A$ we have $\|T\|<\infty$ by uniform boundedness. Hence $T_{j} \stackrel{(s o)}{\longrightarrow} T$ and $T \in E$.

THEOREM A.4.2. The (so) closed convex hull of any (so) compact set $E \subset B(A, A)$ is (so) compact.

Proof. In the (so) topology we have: $E$ compact $\Rightarrow E$ bounded $\Rightarrow \operatorname{co}[E]$ bounded $\Rightarrow \operatorname{co}[E:(s o)]$ bounded. Hence, by A.4.1, co[E: $(s o)]$ is complete. But in any locally convex space, closed convex spans of compact sets are totally bounded (precompact), as shown in [4], p. 8081. Hence $\operatorname{co}[E:(s o)]$ is compact.

An alternative proof is sketched in [7], p. 511, exercises 1-3.

\section{BIBLIOGRAPHY}

1. F. T. Birtel, Banach algebras of multipliers, Duke Math. J. 28 (1961), 203-212.

2. Isomorphisms and isometric multipliers, Proc. Amer. Math. Soc. 13 (1962), 204-210. 
3. F. Bohnenblust and S. Karlin, Geometrical properties of the unit sphere of Banach algebras, Ann. of Math. (2) 62 (1955), 217-229.

4. N. Bourbaki, Espaces Vectoriels Topologiques, Ch. I-II, Hermann et Cie, Paris, 1953.

5. - Integration, Ch. I-IV, Hermann et Cie, Paris, 1952.

6. C. Chevalley, Theory of Lie groups, Vol. I, Princeton Univ. Press, Princeton, N. J., 1946.

7. N. Dunford and J. Schwartz, Linear Operators (Part I), Interscience, New York, 1958.

8. F. P. Greenleaf, Norm decreasing homomorphisms on group algebras, Pacific J. Math. 15 (1965), 1187-1219.

9. K. Hoffman, Banach spaces of analytic functions, Prentice-Hall, Englewood Cliffs, N. J., 1962.

10. I. Kaplansky, Dual rings, Ann. of Math. 49 (1948), 689-701.

11. L. Loomis, Introduction to abstract harmonic analysis, Van Nostrand, New York, 1952.

12. D. Montgomery and L. Zippin, Topological transformation groups, Interscience, New York, 1955.

13. L. Pontryagin, Topological Groups, Princeton Univ. Press, Princeton, N. J., 1946. 14. C. E. Rickart, General theory of Banach algebras, Van Nostrand, New York, 1960. 15. M. A. Rieffel, A characterization of commutative group algebras and measure algebras, $\mathrm{Ph}$. D. dissertation, Columbia Univ., 1963.

16. A. Weil, L'Integration dans les groupes topologiques et ses applications, Hermann et Cie, Paris, 1953.

17. J. G. Wendel, On isometric isomorphisms of group algebras, Pacific J. Math. 1 (1951), 289-311.

18. 2 (1952), 251-261.

Received November 13, 1964. This paper is a portion of the author's doctoral dissertation submitted to Yale University in 1963. The author is indebted to Professor C. E. Rickart, who directed this dissertation, and also to Professors S. Sakai and F. Birtel, for their advice and encouragement. This research was carried out while the author held an NSF Cooperative Fellowship at Yale University; it was prepared for publication with the assistance of Air Force research grant: AFOSR-407-63, and with the partial support of NSF grant: NSF-GP 4020.

University of California, Berkeley 


\section{PACIFIC JOURNAL OF MATHEMATICS}

\section{EDITORS}

H. SAMELSON

Stanford University

Stanford, California

R. M. BLUMENTHAL

University of Washington

Seattle, Washington 98105

\section{*J. DugundJI}

University of Southern California Los Angeles, California 90007

RICHARD ARENS

University of California

Los Angeles, California 90024

\section{ASSOCIATE EDITORS}

E. F. BECKENBACH
F. WOLF

K. YosIDA

\section{SUPPORTING INSTITUTIONS}

\author{
UNIVERSITY OF BRITISH COLUMBIA \\ CALIFORNIA INSTITUTE OF TECHNOLOGY \\ UNIVERSITY OF CALIFORNIA \\ MONTANA STATE UNIVERSITY \\ UNIVERSITY OF NEVADA \\ NEW MEXICO STATE UNIVERSITY \\ OREGON STATE UNIVERSITY \\ UNIVERSITY OF OREGON \\ OSAKA UNIVERSITY \\ UNIVERSITY OF SOUTHERN CALIFORNIA
}

\author{
STANFORD UNIVERSITY \\ UNIVERSITY OF TOKYO \\ UNIVERSITY OF UTAH \\ WASHINGTON STATE UNIVERSITY \\ UNIVERSITY OF WASHINGTON \\ AMERICAN MATHEMATICAL SOCIETY \\ CHEVRON RESEARCH CORPORATION \\ TRW SYSTEMS \\ NAVAL ORDNANCE TEST STATION
}

Mathematical papers intended for publication in the Pacific Journal of Mathematics should be typewritten (double spaced). The first paragraph or two must be capable of being used separately as a synopsis of the entire paper. It should not contain references to the bibliography. Manuscripts may be sent to any one of the four editors. All other communications to the editors should be addressed to the managing editor, Richard Arens at the University of California, Los Angeles, California 90024 .

50 reprints per author of each article are furnished free of charge; additional copies may be obtained at cost in multiples of 50 .

The Pacific Journal of Mathematics is published monthly. Effective with Volume 16 the price per volume (3 numbers) is $\$ 8.00$; single issues, $\$ 3.00$. Special price for current issues to individual faculty members of supporting institutions and to individual members of the American Mathematical Society: $\$ 4.00$ per volume; single issues $\$ 1.50$. Back numbers are available.

Subscriptions, orders for back numbers, and changes of address should be sent to Pacific Journal of Mathematics, 103 Highland Boulevard, Berkeley 8, California.

Printed at Kokusai Bunken Insatsusha (International Academic Printing Co., Ltd.), No. 6, 2-chome, Fujimi-cho, Chiyoda-ku, Tokyo, Japan.

PUBLISHED BY PACIFIC JOURNAL OF MATHEMATICS, A NON-PROFIT CORPORATION

The Supporting Institutions listed above contribute to the cost of publication of this Journal, but they are not owners or publishers and have no responsibility for its content or policies.

* Paul A. White, Acting Editor until J. Dugundji returns. 


\section{Pacific Journal of Mathematics}

\section{Vol. 18, No. 2 \\ April, 1966}

Alexander V. Arhangelskii, On closed mappings, bicompact spaces, and a problem of P. Aleksandrov .............................. 201

A. K. Austin, A note on loops . . . . . . . . . . . . . . . . . . . . . . . . . . . . 209

Lawrence Peter Belluce and William A. Kirk, Fixed-point theorems for families of contraction mappings...................... 213

Luther Elic Claborn, Every abelian group is a class group ............ 219

Luther Elic Claborn, A note on the class group .................. 223

Robert Stephen De Zur, Point-determining homomorphisms on multiplicative semi-groups of continuous functions............. 227

Raymond William Freese, A convexity property ................ 237

Frederick Paul Greenleaf, Characterization of group algebras in terms of their translation operators ......................... 243

Andrzej Hulanicki, On the spectral radius of hermitian elements in group algebras....................................... 277

Michael Bahir Maschler and Bezalel Peleg, A characterization, existence proof and dimension bounds for the kernel of a game ............ 289

Yiannis (John) Nicolas Moschovakis, Many-one degrees of the predicates

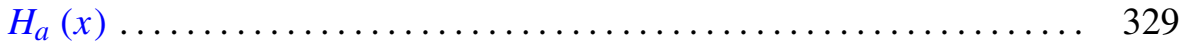

G. O. Okikiolu, $n$th order integral operators associated with Hilbert transforms.

C. E. Rickart, Analytic phenomena in general function algebras ... 361

K. N. Srivastava, On an entire function of an entire function defined by Dirichlet series

Paul Elvis Waltman, Oscillation criteria for third order nonlinear differential equations .......................... 